\title{
Viabilities of seismic ray impedance and elastic impedance for hydrocarbon-sand discrimination
}

\author{
Feng Zhang ${ }^{1}$, Yanghua Wang ${ }^{2}$, and Xiang-Yang $\mathrm{Li}^{3}$
}

\begin{abstract}
Theoretical evaluation of the elastic impedance (EI) and the ray impedance $(\mathrm{RI})$ reveals that RI has a more reliable value range and is less sensitive to noise than EI. We devised a new measurement R̃I to estimate the ray impedance from elastic impedance derived by existing techniques. The recovered R̃ was expressed in the form of a normalization of EI. It solved the range variability problem of EI and had the same interpretation capability as RI. In addition, reflection coefficients represented by RI showed good agreement with the Zeoppritz equation even at postcritical angle of incidence. Tests of these three attributes (RI, RI, and EI) were performed on the log data of three different
\end{abstract}

types of reservoir: a typical Class III marine gas-sand, a Class I tight gas-sand, and a Class II oil-bearing sand in thin sand-mud interbedded layers. Although the crossplots of EI against acoustic impedance (AI) showed visually similar characteristics for a gas-sand as that of RI, based on the linear/quadratic discrimination analysis, RI appeared to be more applicable than EI for characterizing gas sands, especially tight gas sands. R̃I, estimated from EI, had a comparable value range to the AI, and retained the interpretation ability of the original RI. Application on real seismic data showed that existing EI inversion results could be improved straightforwardly by means of the introduced transformation.

\section{INTRODUCTION}

Seismic impedance is an intrinsic physical property of subsurface media. The acoustic impedance (AI) is expressed as the product of bulk density and P-wave velocity

$$
\mathrm{AI}_{i}=\alpha_{i} \rho_{i},
$$

where $i$ refers to the $i$ th medium layer, $\alpha_{i}$ and $\rho_{i}$ are the P-wave velocity and the density of the layer. It is related to the P-wave normal incidence reflection by

$$
R_{i}(0)=\frac{\mathrm{AI}_{i+1}-\mathrm{AI}_{i}}{\mathrm{AI}_{i+1}+\mathrm{AI}_{i}} \approx \frac{1}{2} \ln \frac{\mathrm{AI}_{i+1}}{\mathrm{AI}_{i}} .
$$

As a generalization of AI, the elastic impedance (EI) is defined to delineate the medium property of the reflection from nonnormal Pwave incidence (Connolly, 1999)

$$
\mathrm{EI}_{i}=\alpha_{i} \rho_{i}\left(\alpha_{i}^{\tan ^{2} \theta} \beta_{i}^{-8 K \sin ^{2} \theta} \rho_{i}^{-4 K \sin ^{2} \theta}\right)
$$

where $\beta_{i}$ is S-wave velocity of the medium layer, $\theta$ is the average angle of the incidence angle $\theta_{i}$, and the emanating/transmission angle $\left(\theta_{i+1}\right.$ or $\left.\theta_{e}\right)$, and $K=\beta^{2} / \alpha^{2}$ is assumed to be constant in the EI definition.

Elastic impedance inversion has been applied successfully in many cases to characterize the hydrocarbon reservoir (Connolly, 1999; Avseth, 2005). EI inversion is also favored by the industry because, given an angle of incidence, it can be performed on prestack seismic data similarly to the poststack AI inversion.

A practical limitation of EI is that its value range varies dramatically with the angle of incidence. This range variability problem could cause a difficulty in using EI for reservoir characterization. A normalized form of EI is proposed in Whitcombe (2002) to solve the range variability problem

\footnotetext{
Manuscript received by the Editor 19 July 2011; revised manuscript received 9 December 2011; published online 14 June 2012.

${ }^{1}$ China University of Petroleum, Department of Geophysical Prospecting, State Key Laboratory of Petroleum Resource and Prospecting, Beijing, China; Imperial College London, Department of Earth Science and Engineering, Center for Reservoir Geophysics, London, U. K. E-mail: f.zhang717@googlemail.com.

${ }^{2}$ Imperial College London, Department of Earth Science and Engineering, Center for Reservoir Geophysics, London, U. K. E-mail: yanghua.wang @imperial ac.uk.

${ }^{3}$ China University of Petroleum, Department of Geophysical Prospecting, State Key Laboratory of Petroleum Resource and Prospecting, Beijing, China; University of Edinburgh, British Geological Survey, Edinburgh, U. K. E-mail: xyl@bgs.ac.uk.

(C) 2012 Society of Exploration Geophysicists. All rights reserved.
} 


$$
\mathrm{EI}_{i}^{\mathrm{norm}}(\theta)=\alpha_{i} \rho_{i}\left(\left(\frac{\alpha_{i}}{\alpha_{0}}\right)^{\tan ^{2} \theta}\left(\frac{\beta_{i}}{\beta_{0}}\right)^{-8 K \sin ^{2} \theta}\left(\frac{\rho_{i}}{\rho_{0}}\right)^{-4 K \sin ^{2} \theta}\right)
$$

where $\alpha_{0}, \beta_{0}$, and $\rho_{0}$ are the reference values for the subsurface interval of interest.

The ray impedance (RI) is defined along a seismic wave propagation ray path honoring Snell's law. It not only includes the P- to $\mathrm{S}$-wave conversion, but also considers the $\mathrm{P}$-wave transmission. In this sense, it is a generalization of the elastic impedance, and represents the physical properties of continuous subsurface media. At the $i$ th interface, the ray impedance is defined by (Wang, 2003)

$$
\begin{aligned}
\mathrm{RI}_{i}\left(p\left(\theta_{i}\right)\right) & =\frac{\alpha_{i} \rho_{i}}{\sqrt{1-\alpha_{i}^{2} p^{2}}}\left(1-\beta_{i}^{2} p^{2}\right)^{2(r+2)} \\
& =\frac{\alpha_{i} \rho_{i}}{\cos \theta_{i}}\left(1-\frac{\beta_{i}^{2}}{\alpha_{i}^{2}} \sin ^{2} \theta_{i}\right)^{2(r+2)},
\end{aligned}
$$

where $p=\sin \theta_{i} / \alpha_{i}$ is the ray parameter or horizontal slowness, and $r=(\Delta \rho / \rho) /(\Delta \beta / \beta)$ is assumed to be constant, with $\Delta \rho$ and $\Delta \beta$ as the different values of density and S-wave velocity across the interface, respectively. This definition is derived based on Wang's (1999) quadratic approximation of the reflection coefficient. A similar impedance definition in the ray-parameter domain is proposed by Santos and Tygel (2004) based on Aki and Richards (1980) linearized approximation, and VerWest (2004) based on Bortfeld's (1961) approximation.

In this paper, we first evaluate the derivation and interpretation capability of the elastic impedance and the ray impedance using three single-interface models. Parameters of each model are extracted from the real logs having their respective reservoir type (Table 1). We show that at the precritical angle, elastic impedance has generally a good accuracy in representing the reflection coefficient, from which it is derived. The ray impedance can approximate the reflectivity not only at the precritical angle, but also at the postcritical angle, because it considers the incident and transmission angles of an adjacent subsurface interface. EI and RI lead to a similar interpretation of a hydrocarbon-bearing sand over the range of small and middle angles of incidence; while at a wide incident angle, the ray impedance is a better approximation than elastic impedance. The significantly varying value range of EI creates inconvenience in displaying EI and AI logs simultaneously; therefore EI usually needs to be normalized (Whitcombe, 2002). Furthermore, because of this property, nonnormalized EI with inaccurate values of
$K$ produce very different results from that with $K$ in true value, not only in the absolute values, but also in the contrast between neighboring EI samples. Evaluations show that, compared with the RI function (equation 5), EI function (equation 3) is more sensitive to errors from their respective parameter that assumed to be constant.

Instead of comparing the elastic impedance with the ray impedance, we introduce an analytic formula by which RI can be directly estimated from EI, or vice versa. The physical meaning of the original impedance approximations remains during this transformation. Estimation of RI from EI by means of this formula acts as an appropriate normalization of EI. The new proposed ray impedance estimate (R̃) retains the advantages of EI and RI: high accuracy in representing the reflectivity and good interpretation capability. Finally, we calculate these three impedance quantities for the log data from three types of reservoirs, and compare their properties. The introduced equation shows its practical value by straightforwardly improving the EI inversion result .

\section{DERIVATION OF ELASTIC AND RAY IMPEDANCE}

At the $i$ th interface, the PP-wave reflection coefficients at the angle of incidence $\theta$ can be expressed in a recursive form of the elastic impedance above and below the interface by

$$
R_{i}(\theta)=\frac{\mathrm{EI}_{i+1}(\theta)-\mathrm{EI}_{i}(\theta)}{\mathrm{EI}_{i+1}(\theta)+\mathrm{EI}_{i}(\theta)} \approx \frac{1}{2} \ln \frac{\mathrm{EI}_{i+1}(\theta)}{\mathrm{EI}_{i}(\theta)} .
$$

The elastic impedance could be derived based on equation 6 , if the well-known linearized form of the Zoeppritz equations is used (Appendix A)

$$
\begin{aligned}
R_{L(i)}(\theta) \approx & \frac{1}{2}\left(1+\tan ^{2} \theta\right) \frac{\Delta \alpha}{\alpha}-4 \frac{\beta^{2}}{\alpha^{2}} \sin ^{2} \theta \frac{\Delta \beta}{\beta} \\
& +\left[\frac{1}{2}-2 \frac{\beta^{2}}{\alpha^{2}} \sin ^{2} \theta\right] \frac{\Delta \rho}{\rho},
\end{aligned}
$$

where $\alpha, \beta$, and $\rho$ are average value of $\mathrm{P}$-wave velocity, $\mathrm{S}$-wave velocity, and density of two adjacent medium layers; $\Delta \alpha, \Delta \beta$, and $\Delta \rho$ are the difference value of the three elastic parameters across the interface; $\theta$ is the average angle defined above $\left(\theta \approx \theta_{i}\right)$.

The derivation of EI is based on two well-known assumptions.

\begin{tabular}{|c|c|c|c|c|}
\hline & & $V_{\mathrm{P}}(\mathrm{km} / \mathrm{s})$ & $V_{\mathrm{S}}(\mathrm{km} / \mathrm{s})$ & Density $(\mathrm{km} / \mathrm{s}) *(\mathrm{~g} / \mathrm{cc})$ \\
\hline Model 1 (Typical Class III gas-sand) & Upper & 2.886 & 1.016 & 2.271 \\
\hline \multirow[t]{2}{*}{ Model 2 (Tight Class I gas-sand) } & Upper & 4.316 & 2.437 & 2.65 \\
\hline & Lower & 5.3357 & 3.0 & 2.48 \\
\hline
\end{tabular}
The first assumption is that the average angle $\theta$ is constant, implying that the impedance contrast of media above and below the interface is small; thus the angle change across is negligible. The derivation of EI also assumes that parameter $K=\beta^{2} / \alpha^{2}$ is constant. EI is

Table 1. Parameters of three single-interface models. 
sensitive to inaccurate values of $K$, a correction procedure is normally required after the estimation of EI (Connolly, 1999). In practice, the optimum approximation of $K$ is estimated over a target interval with limited time/depth instead of the entire sample series.

Based on the linearized quadratic approximation of reflection coefficients in the ray-parameter domain (Wang, 1999)

$$
R_{L Q(i)}(p)=\operatorname{Rf}-2 \frac{\Delta \mu}{\rho} p^{2},
$$

the ray impedance (equation 4) is derived using the following expression (Appendix B)

$$
R_{L Q(i)}(p)=\frac{\mathrm{RI}_{i+1}-\mathrm{RI}_{i}}{\mathrm{RI}_{i+1}+\mathrm{RI}_{i}} \approx \frac{1}{2} \ln \frac{\mathrm{RI}_{i+1}}{\mathrm{RI}_{i}},
$$

where the P-wave-dependent reflection coefficient $\mathrm{Rf}$ is $\mathrm{Rf}=$ $\frac{\rho_{i+1} q_{\alpha(i)}-\rho_{i} q_{\alpha(i+1)}}{\rho_{i+1} q_{\alpha(i)}+\rho_{i} q_{\alpha(i+1)}}$, with $q_{\alpha(i)}=\sqrt{\frac{1}{\alpha_{i}^{2}}-p^{2}}$ and $q_{\alpha(i+1)}=\sqrt{\frac{1}{\alpha_{i+1}^{2}}-p^{2}}$ are the P-wave vertical slownesses; $q_{\alpha}$ and $q_{\beta}$ represent average $\mathrm{P}$-wave and $\mathrm{S}$-wave vertical slownesses, respectively; and $\Delta \mu=\rho_{i+1} \beta_{i+1}^{2}-\rho_{i} \beta_{i}^{2}$ is the contrast in shear modulus.

Because the ray-parameter is constant at different depths along the wave propagation path, RI can be used to describe the continuous subsurface media over an arbitrary depth interval, even at a postcritical angle. The parameter $r=(\Delta \rho / \rho) /(\Delta \beta / \beta)$ is assumed to be constant, and can be adjusted for different ratios of density contrast to shear-wave velocity contrast. In fact, RI is almost inaccurate with values of $r$; beside, RI of larger ray parameters generally gives better discrimination of hydrocarbon reservoir from surrounding rocks, and it has little range difference from the RI with smaller ray parameters or AI (Zhang, 2010).

To compare the accuracy of EI and RI in representing the reflection coefficients, we plot different forms of approximation for the $\mathrm{PP}$-wave reflection coefficients at the angle of incidence from $0^{\circ}$ to $70^{\circ}$ in Figure 1. Three single-interface models are built using well $\log$ data from different types of reservoirs (Table 1). The linearized approximations (equations 7 and 8) have very similar accuracy for the three models at precritical angle. Equation 7 fails to represent the reflectivity in the regions of critical and postcritical angles for Models 2 and 3. The reflectivity calculated from EI (equation 8) agrees well with its original linearized approximation for three models because the derivation of EI is not compromised much on the accuracy of the reflectivity (Appendix A). Although the reflectivity approximated by RI (equation 9) follows the general behavior of the exact Zoeppritz curve even in the region of postcritical angle, it shows deviation from its original approximation at a large angle of incidence.

\section{EVALUATION OF ELASTIC AND RAY IMPEDANCE IN INTERPRETATION}

To evaluate the interpretation ability of the elastic impedance, EI curves of three models are generated within angle of incidence from $0^{\circ}$ to $50^{\circ}$ (Figure 2). Different types of curves indicate EI with different values of $K$ for the overburden shale layer and lower sand layer. In Model 1 because the difference between EI curves increases with angle, elastic impedance provides generally good discrimination of sand from shale. However, even with an optimum K, EIs at large angles show very different performance from
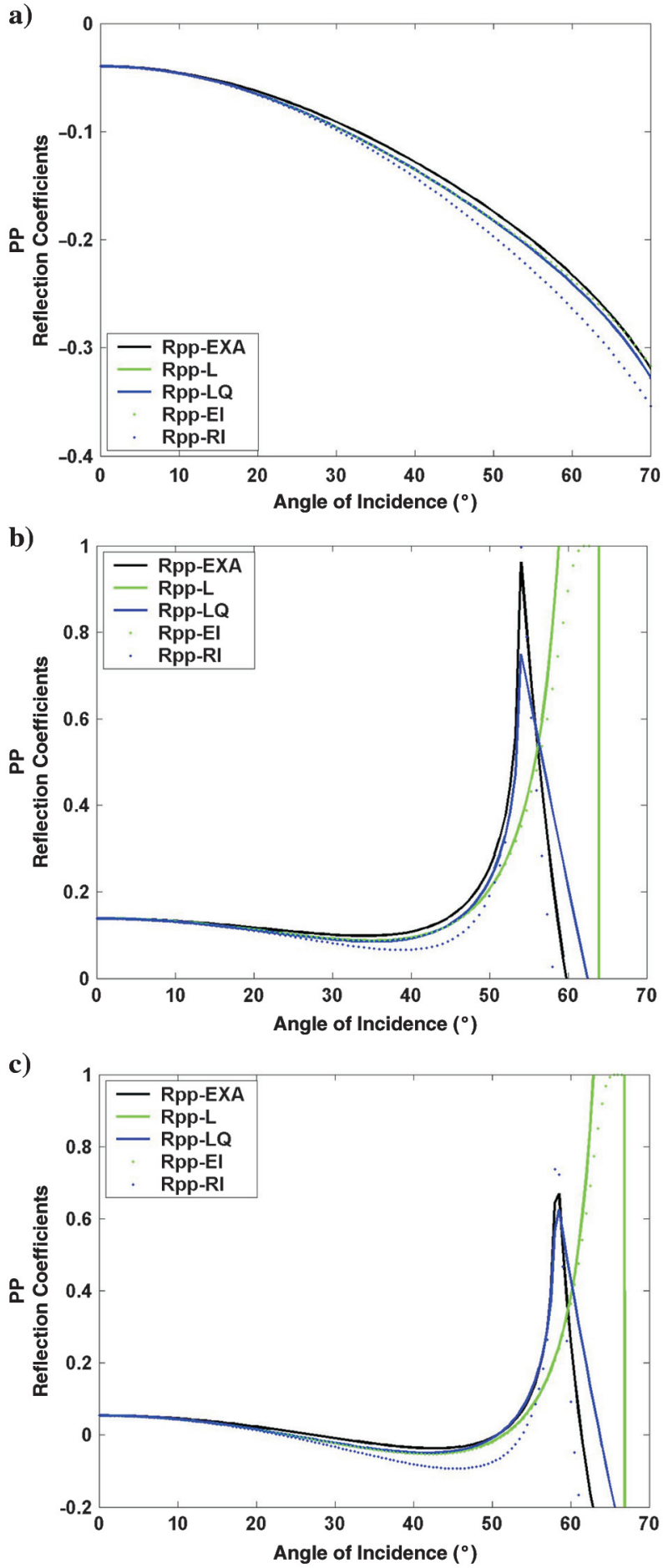

Figure 1. Comparison of different approximations of reflection coefficients (AVO) for three models: (a) Model 1: Typical marine Class III gas-sand; (b) Model 2: Tight Class I gas-sand; and (c) Model 3: Thin sand-mud interbedded layers (oil-bearing sand). The black curves represent the reflection coefficients from the exact Zoeppritz equations. The solid green and blue lines represent the linearized approximations from equations 7 and 8, respectively; the dotted green and blue lines, respectively, show the approximations derived from EI (equation 3) and RI (equation 5). The dotted green curves coincide with the solid green curves in three figures, thus they are not discernable. The dotted/solid green curves also coincide with the solid blue curves in (a). 
a)
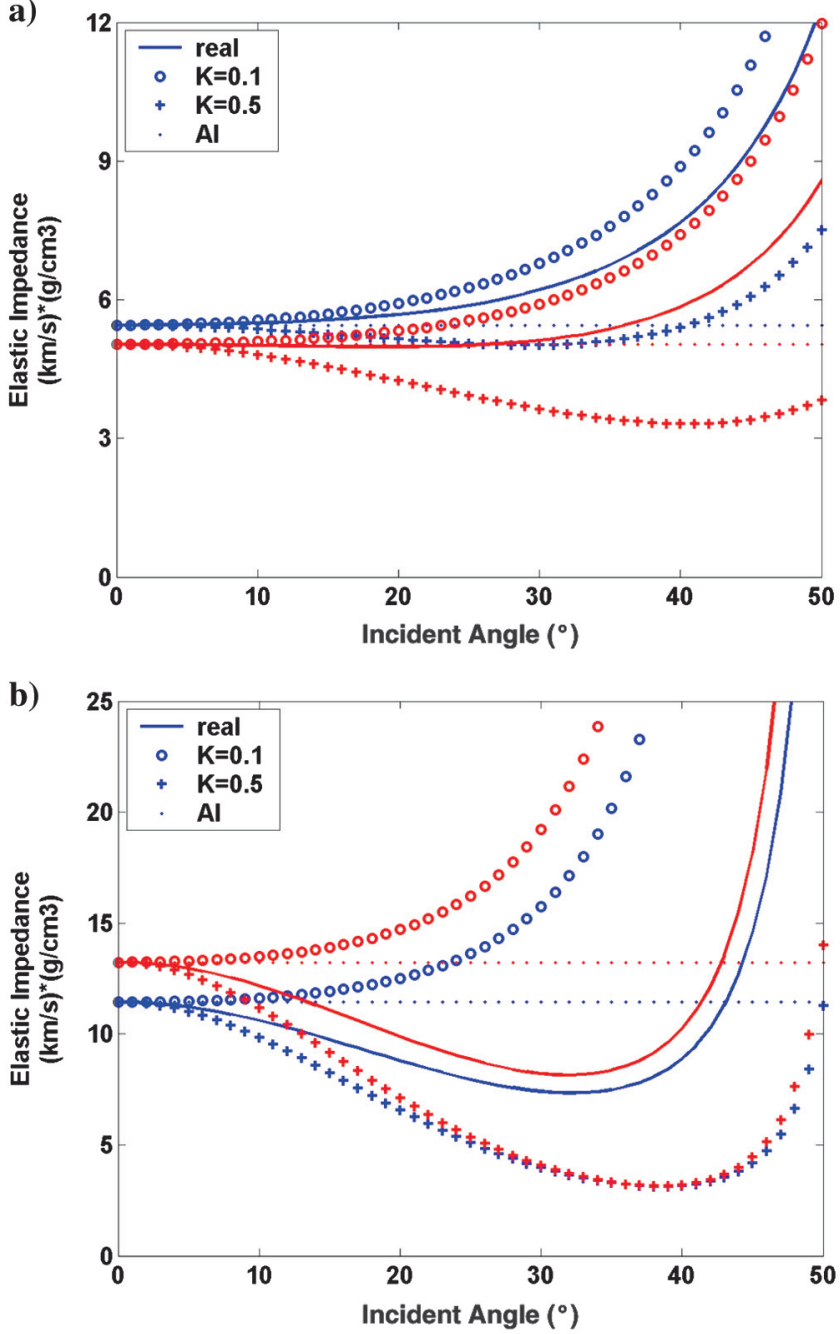

c)

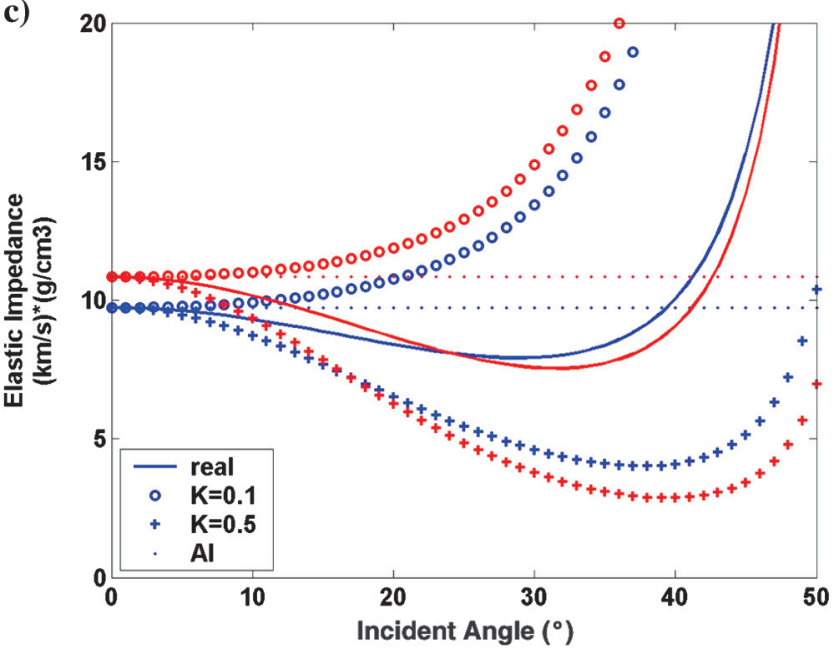

Figure 2. EI with different values of $K$ for (a) Model 1, (b) Model 2, and (c) Model 3: Solid curves are EIs with the real value of $K$ of $0.218,0.317$, and 0.297 , respectively; while dotted curves are EIs with inaccurate values of $K$, which are set to be 0.1 and 0.5 according to the real values. AIs are plotted as the straight dotted lines. The overburden shale layers and lower sand layers are represented in blue and red, respectively. those at small and middle angles in Models 2 and 3: EIs decrease gradually from 12 to $8(\mathrm{~km} / \mathrm{s}) *(\mathrm{~g} / \mathrm{cc})$ when $\theta<40^{\circ}$; but when $\theta>40^{\circ}$, EI values dramatically increase and reach up to $40(\mathrm{~km} / \mathrm{s}) *(\mathrm{~g} / \mathrm{cc})$ when $\theta$ approaches $50^{\circ}$. Given different values of $K$, EI curves vary significantly with each other, not only in their values but also in their shape. Inaccurate $K$ changes not only the contrast between EIs of the two layers, but also the relative relationship between EI and AI (straight dotted lines). This could lead to difficulties in discriminating a hydrocarbon-bearing sand from a shale.

Based on the normalized form of EI in equation 4, if optimized $\alpha_{0}, \beta_{0}$, and $\rho_{0}$ (average values of the elastic constants) are applied, the range variability problem will be resolved efficiently, and the normalized EI becomes less sensitive to an inaccurate $K$ (Figure 3a-3c). However, in Figure $4 a-4 c$, when nonoptimized reference measurements are used (variation at $10 \%$ ), EIs perform differently from the corresponding cases in Figure $3 a-3 c$. In fact, this estimation is sensitive to not only inaccurate values of $K$ but also the normalization reference measurements (Figures 3 and 4). So optimized reference measurements $\left(\alpha_{0}, \beta_{0}\right.$, and $\left.\rho_{0}\right)$ are very important to the accuracy of $\mathrm{EI}^{\mathrm{norm}}$.

Ray impedance curves show gradually upward or downward trends with the increase of the incidence angle (Figure 5a-5c), so a normalization procedure is not necessary after RI is estimated. RIs with different $r$ deviate only slightly from each other. In other words, an inaccurate $r$ has little impact neither on the contrast between RIs of upper and lower layers, nor on the relative relationship between RI and AI. In Model 1, RI with a higher value of incidence (ray-parameter) has a better discrimination of sand from shale than that of smaller incident angle, because the difference between the RIs of shale and sand increases gradually with incident angle (Figure 5a). In the case of Models 2 and 3, the difference between RI and AI grows generally with the increase of incidence angle. Therefore the crossplot of RI against AI is also capable of indicating gas-sand.

Different ray impedance approximations are plotted in Figure 6a to $6 \mathrm{c}$. They are compared with each other in a zoomed scale. In general, Santos and Tygel's approximation has slightly larger values, while VerWest's approximation has slightly smaller value. All of the three approximations show a similar behavior with an increasing angle.

To sum up the above two sections, we now discuss the advantages and disadvantages of elastic impedance and ray impedance. The derivation of elastic impedance preserves certain accuracy of its original reflectivity. Through a series of theoretical evaluations, we show that EI can generally discriminate reservoir-bearing sand when the incident is smaller than $40^{\circ}$, and the target of interest is local. However, elastic impedance needs to be used carefully because it is sensitive to the inaccurate values of $K$ and its range variability problem at large angle of incidence.

Except for a slight variation in impedance values, inaccurate values of the parameter $r$ have little impact on the performance of ray impedance in characterizing hydrocarbon reservoir. Over the wide range of the incidence angle, the ray impedance has a comparable value to AI, and RI with larger ray-parameters show generally better discrimination of sand from shale. Therefore, the ray impedance is more applicable than elastic impedance for interpretation. A disadvantage of RI is that there is a compromise of accuracy in representing reflectivity during the derivation of RI. 


\section{TRANSFORMATION FROM EI TO RI}

RI, compared to EI, has more stable value range and insensitivity to errors in the parameter $r$; however, it is still a new attribute to the industry. Geophysicists have become accustomed to generating seismic angle gathers using previously developed techniques. For
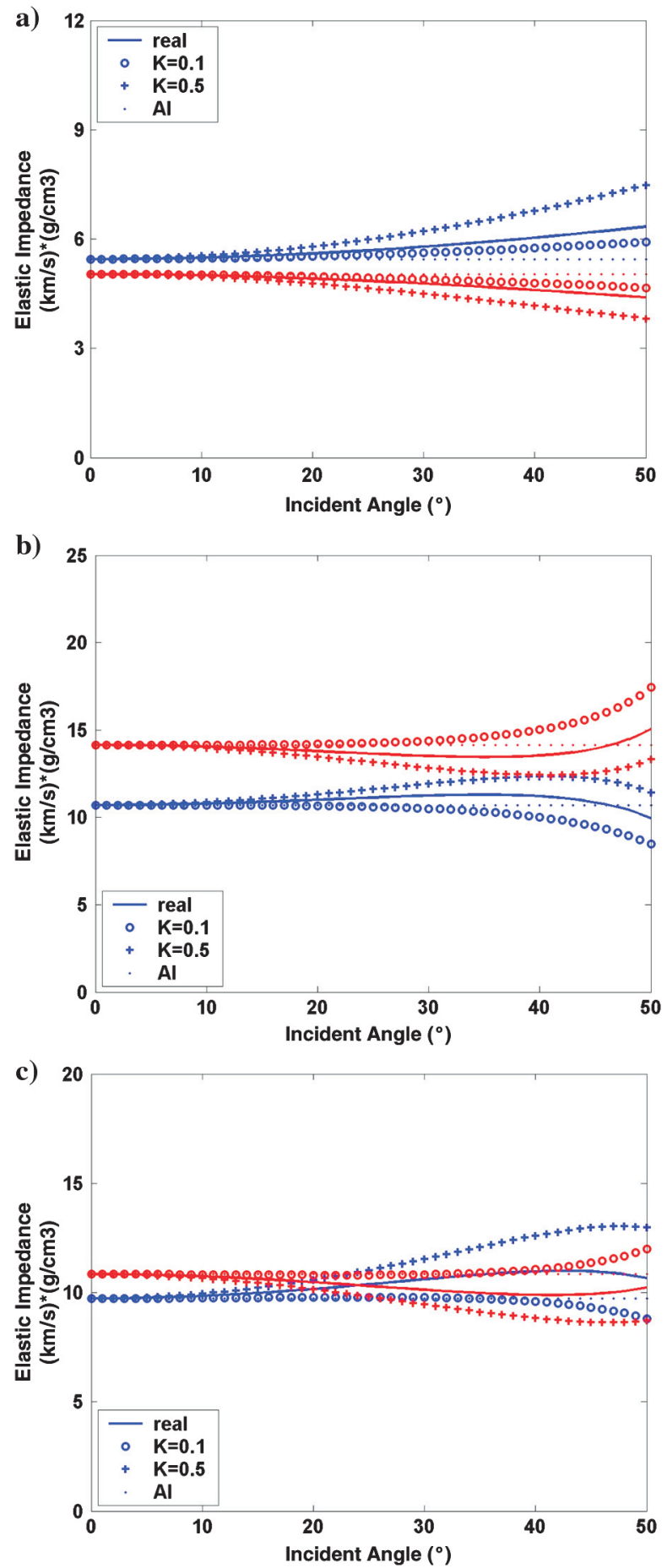

Figure 3. Normalized EI with different values of $K$ for three models: (a-c) Optimally normalized EI with different values of $K$ for Model 1 (left), Model 2 (middle), and Model 3 (right). example, real angle-domain PP-wave CIP gathers can be produced during prestack depth migration (Xu et al., 2001; Sava and Fomel, 2003). EI is then inverted from the seismic data within a range of incident angles. Therefore, beside quantitative comparison of EI with RI, it is meaningful to build a direct link; for example, a quantitative transformation formula, between these two valuable
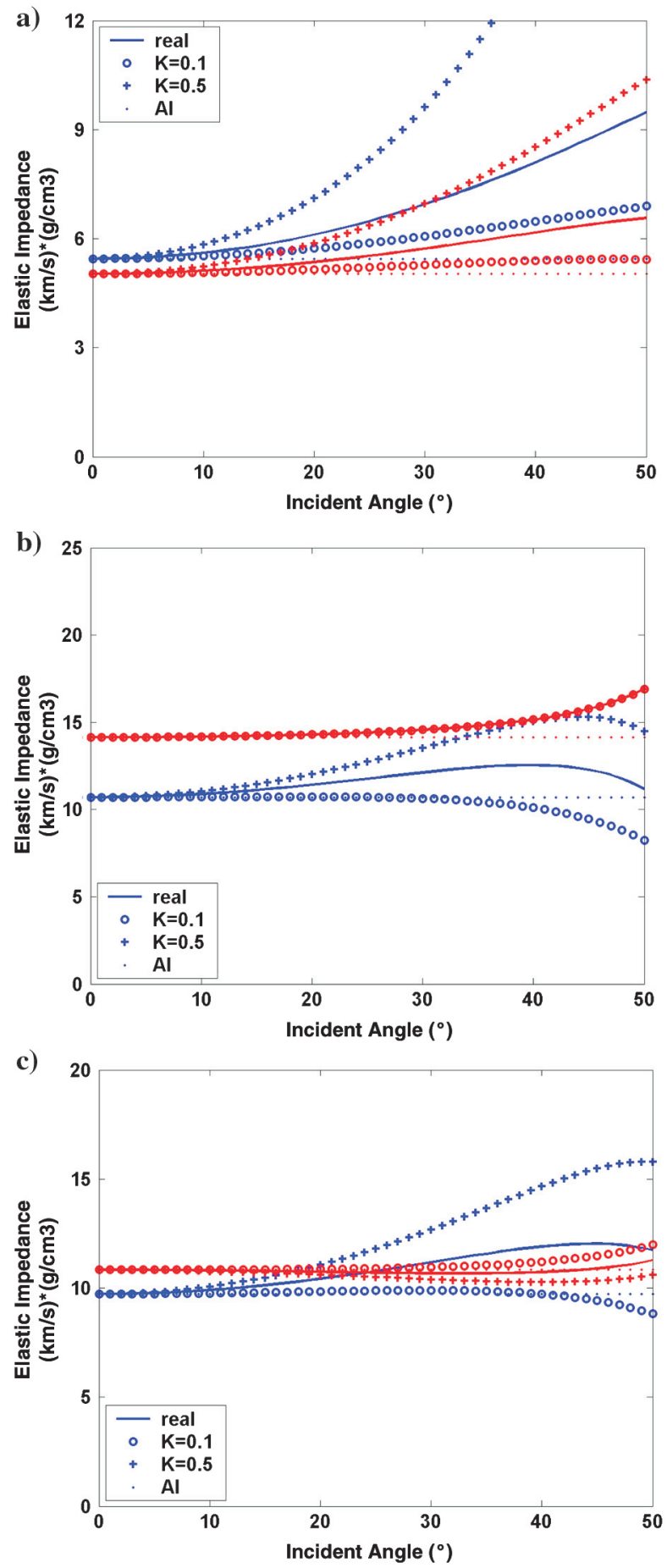

Figure 4. Normalized EI with different values of $K$ for three models: (a-c) Nonoptimally normalized EI with different values of $K$ for Model 1 (left), Model 2 (middle), and Model 3 (right). 
attributes, so that existing EI inversion results could be improved straightforwardly by means of such a transformation.

We propose an analytic formula to estimate the ray impedance from the elastic impedance. This estimated ray impedance is
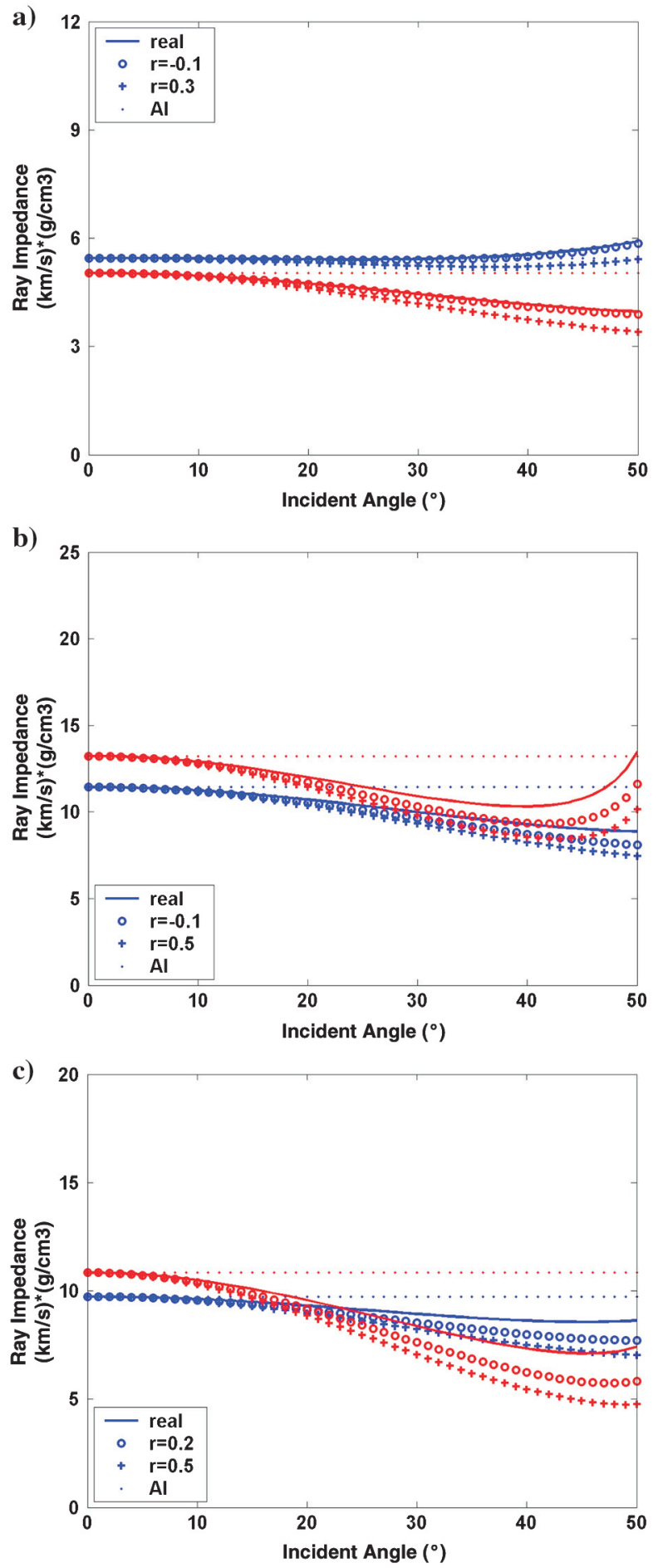

Figure 5. RI with different values of $r$ for (a) Model 1, (b) Model 2, and (c) Model 3. The real value of $r$ is $-0.155,-0.32$, and -0.164 , respectively, which are calculated using the model parameters; while the inaccurate values of $r$ is set in a range according to the real values. denoted as RI, and represents a combination of the above two attributes (EI and RI) which can be expressed in the form of a normalization to EI.

In above sections, we showed that $\operatorname{EI}(\theta)$ and $\operatorname{RI}(p(\theta))$ have different performances in hydrocarbon-sand characterization, though
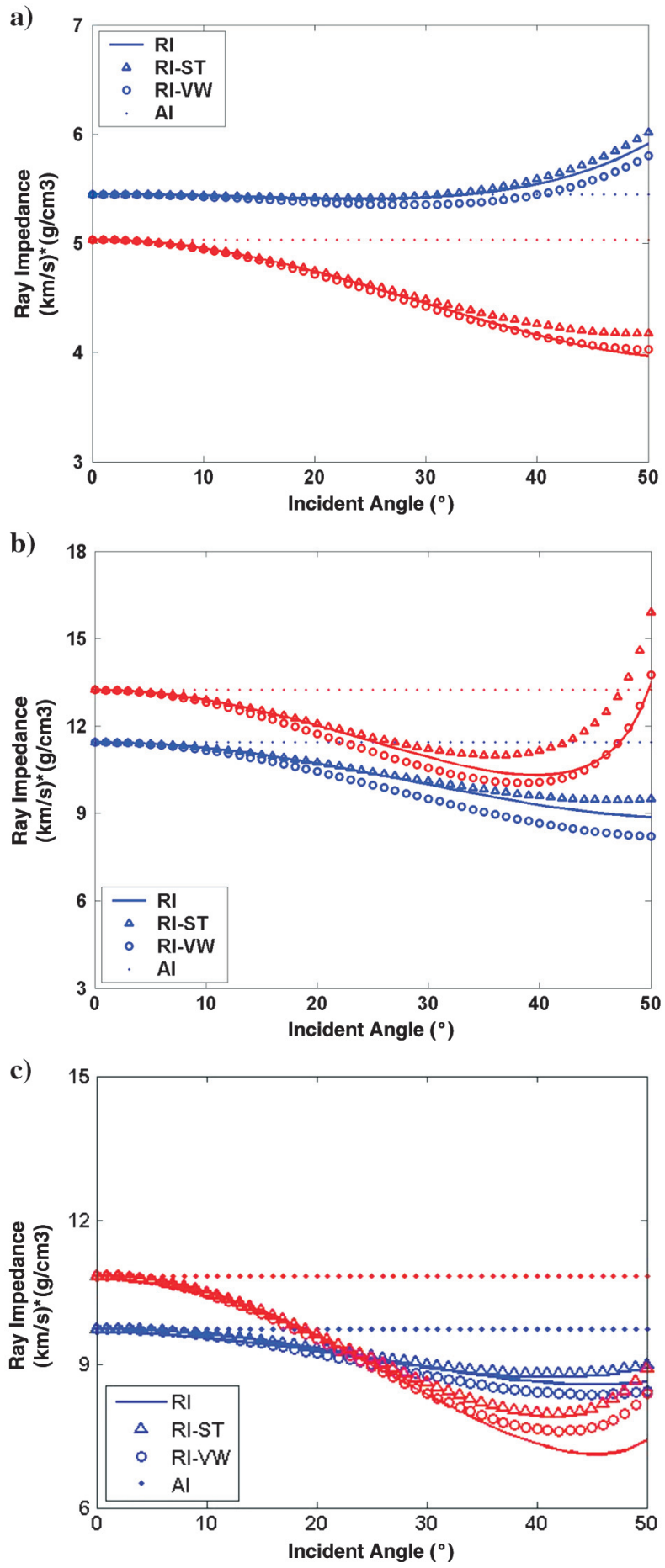

Figure 6. Different forms of ray-impedances for (d) Model 1, (e) Model 2, and (f) Model 3 are compared: Solid curves are ray impedance in equation 5. Symbols $\bigcirc$ and $\triangle$ represents ray impedance approximations in Santos and Tygel (2004) and VerWest (2004), respectively. 
they are derived from the reflectivity with similar accuracy. Estimation of RI from EI is not easy. It should fulfill two requirements: first, the physical meaning of the original measurement (EI), for example the angle domain must retain; second, the reflectivity calculated using the new estimate should have an appropriate accuracy.

At the $i$ th interface, we use a general expression to represent the two forms of PP-wave reflection coefficients in equations 7 and 8

$$
R \approx \mathrm{Rf}+\mathrm{Rg} \approx \frac{1}{2} \ln \frac{I_{f(i+1)} I_{g(i+1)}}{I_{f(i)} I_{g(i)}},
$$

where $\mathrm{Rf}$ and $\mathrm{Rg}$ are the $\mathrm{P}$-wave dependent term and S-wave dependent term, respectively; If and Ig are their respective impedance term; $\mathrm{Rf}$ and $\mathrm{Rg}$ are also referred to as the fluid-fluid term and the rigidity term in the pore-fluid substitution modeling (Hilterman, 2001). The first two approximations are

$$
\operatorname{Rf}_{L(i)}=\frac{1}{2}\left[\left(1+\tan ^{2} \theta\right) \frac{\Delta \alpha}{\alpha}+\frac{\Delta \rho}{\rho}\right] \approx \frac{1}{2} \ln \frac{\mathrm{EI}_{f(i+1)}}{\mathrm{EI}_{f(i)}}
$$

and

$$
\begin{aligned}
\operatorname{Rf}_{L Q(i)} & =\frac{\rho_{i+1} q_{\alpha(i)}-\rho_{i} q_{\alpha(i+1)}}{\rho_{i+1} q_{\alpha(i)}+\rho_{i} q_{\alpha(i+1)}} \approx \frac{1}{2} \ln \left(\frac{\alpha_{i+1} \rho_{i+1}}{\cos \theta_{i+1}} / \frac{\alpha_{i} \rho_{i}}{\cos \theta_{i}}\right) \\
& \approx \frac{1}{2} \ln \frac{\mathrm{RI}_{f(i+1)}}{\mathrm{RI}_{f(i)}},
\end{aligned}
$$

respectively, where

$$
\mathrm{EI}_{f(i)}=\alpha_{i}^{1+\tan ^{2} \theta} \rho_{i}
$$

and

$$
\mathrm{RI}_{f(i)}=\frac{\alpha_{i} \rho_{i}}{\cos \theta_{\mathrm{i}}}
$$

Based on equations 13 and 14 , estimation of $\tilde{\mathrm{RI}}_{f(i)}$ from $\mathrm{EI}_{f(i)}$ seems to be straightforward $\left(\tilde{\mathrm{RI}}_{f(i)} \approx \mathrm{EI}_{f(i)} \frac{\alpha_{i}^{-\tan ^{2} \theta}}{\cos \theta_{i}}\right)$. However, this transformation is meaningful only if the reflectivities represented by $\tilde{\mathrm{RI}}_{f}$ and $\mathrm{EI}_{f}$ (i.e., equations 11 and 12) have similar accuracy. Using the equation B9 (see Appendix B)

$$
\tan \frac{\Delta \theta}{2} \approx \frac{\Delta \theta}{2} \approx \frac{1}{2} \tan \theta \frac{\Delta \alpha}{\alpha}
$$

we rewrite the fluid-fluid term in equation 12 as

$$
\begin{aligned}
\operatorname{Rf}_{L Q(i)} & \approx \frac{1}{2}\left[\frac{\Delta \alpha}{\alpha}+\frac{\Delta \rho}{\rho}+\frac{\Delta \cos \theta}{\cos \theta}\right] \\
& \approx \frac{1}{2}\left[\frac{\Delta \alpha}{\alpha}+\frac{\Delta \rho}{\rho}+\Delta \theta \tan \theta\right] \\
& \approx \frac{1}{2}\left[\left(1+\tan ^{2} \theta\right) \frac{\Delta \alpha}{\alpha}+\frac{\Delta \rho}{\rho}\right] \\
& =\operatorname{Rf}_{L(i)} .
\end{aligned}
$$

Combining equations 11,12 , and 15 , we have

$$
\frac{1}{2} \ln \frac{\mathrm{EI}_{f(i+1)}}{\mathrm{EI}_{f(i)}} \approx \frac{1}{2} \ln \frac{\mathrm{RI}_{f(i+1)}}{\mathrm{RI}_{f(i)}}
$$

Now, $\tilde{\mathrm{RI}}_{f}$ could be estimated from $\mathrm{EI}_{f}$ according with the expressions 13 and 14

$$
\begin{aligned}
\frac{1}{2} \ln \frac{\mathrm{EI}_{f(i+1)}}{\mathrm{EI}_{f(i)}} & \approx \frac{1}{2} \ln \frac{\tilde{\mathrm{RI}}_{f(i+1)}}{\tilde{\mathrm{RI}}_{f(i)}} \\
& \approx \frac{1}{2} \ln \left(\frac{\mathrm{EI}_{f(i+1)} \alpha_{i+1}^{-\tan ^{2} \theta}}{\cos \theta_{i+1}} / \frac{\mathrm{EI}_{f(i)} \alpha_{i}^{-\tan ^{2} \theta}}{\cos \theta_{i}}\right) .
\end{aligned}
$$

Therefore, the fluid term of estimated ray impedance is indeed

$$
\tilde{\mathrm{RI}}_{f(i)} \approx \mathrm{EI}_{f(i)} \frac{\alpha_{i}^{-\tan ^{2} \theta}}{\cos \theta_{i}}
$$

In Figure 7, curves of $\mathrm{Rf}_{L}$ and $\mathrm{Rf}_{L Q}$ have nearly the same accuracy, $\mathrm{Rf}$ represented by $\mathrm{RI}_{f}$ is very close to its original approximation, Rf represented by $\mathrm{EI}_{f}$ has deviation from the other reflectivites. However, the reflectivity curve derived from $\tilde{\mathrm{RI}}_{f}$ agrees with the $\mathrm{Rf}$ curve derived from $\mathrm{EI}_{f}$ over the whole range of angles of incidence.

The rigidity terms used to derive EI and RI are

$$
\begin{aligned}
\operatorname{Rg}_{L(i)} & =-\frac{1}{2}\left[8 K \sin ^{2} \theta \frac{\Delta \beta}{\beta}+4 K \sin ^{2} \theta \frac{\Delta \rho}{\rho}\right] \\
& \approx \frac{1}{2} \ln \frac{\mathrm{EI}_{\mathrm{g}(i+1)}}{\mathrm{EI}_{g(i)}},
\end{aligned}
$$

and

$$
\operatorname{Rg}_{L Q(i)}=-2 \frac{\Delta \mu}{\rho} p^{2} \approx \frac{1}{2} \ln \frac{\mathrm{RI}_{g(i+1)}}{\mathrm{RI}_{g(i)}}
$$

with

$$
\mathrm{EI}_{g(i)}=\beta_{i}^{-8 K \sin ^{2} \theta} \rho_{i}^{-4 K \sin ^{2} \theta}
$$

and

$$
\mathrm{RI}_{g(i)}=\cos ^{4(r+2)} \varphi_{i}
$$

However, it is not appropriate to derive $\tilde{\mathrm{RI}}_{g}$ in a method similar to equation 16 because

$$
\frac{1}{2} \ln \frac{\mathrm{EI}_{g(i+1)}}{\mathrm{EI}_{g(i)}} \neq \frac{1}{2} \ln \frac{\mathrm{RI}_{g(i+1)}}{\mathrm{RI}_{g(i)}}
$$

This difference is caused by the assumption made in the derivation of $\operatorname{Rg}_{i}\left(p\left(\varphi_{i}\right)\right)$ (see Appendix B). To establish a comparable equation between equations 19 and 20, we first make an approximation of $\operatorname{Rg}_{L Q}$ based on expression B4 by 
$\operatorname{Rg}_{L Q(i)} \approx \frac{1}{2} \ln \frac{\mathrm{RI}_{g(i+1)}^{\prime}}{\mathrm{RI}_{g(i)}^{\prime}} \approx \frac{1}{2} \ln \frac{\beta_{i+1}^{-4 \tan ^{2} \varphi(r+2)}}{\beta_{i}^{-4 \tan ^{2} \varphi(r+2)}}$

This approximation now has very similar accuracy to expressions 19
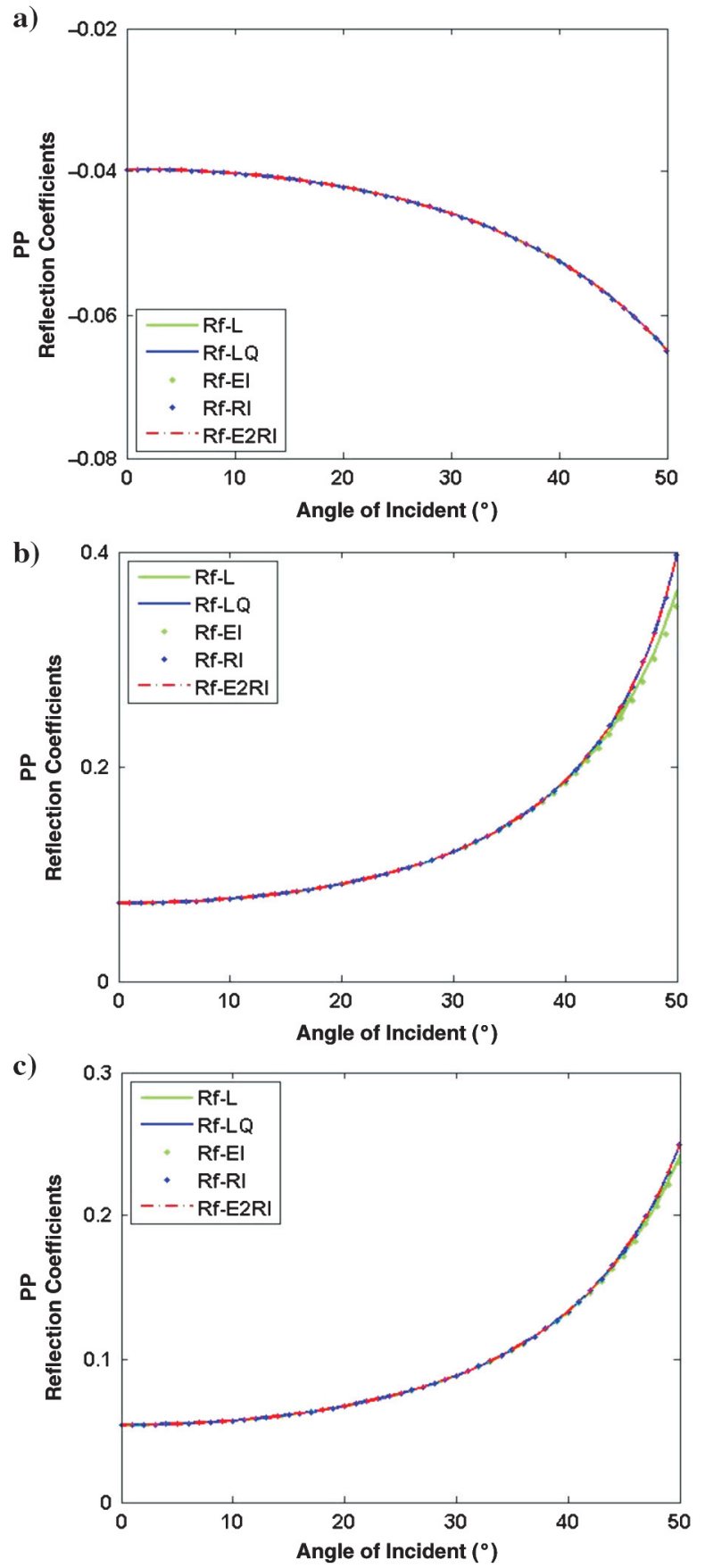

Figure 7. Comparison of different forms of Rg curves for (a) Model 1, (b) Model 2, and (c) Model 3. Solid green and blue curves represent the linearized approximations from equation 19 and equation 20, respectively. The dotted green and blue lines, respectively, show the approximations derived from $\mathrm{EI}_{g}$ (equation 21 and $\mathrm{RI}_{g}$ (equation 22. Dashed red curves are the reflectivity derived from $\mathrm{RI}_{g}$.

$$
\frac{1}{2} \ln \frac{\mathrm{EI}_{g(i+1)}}{\mathrm{EI}_{g(i)}} \approx \frac{1}{2} \ln \frac{\mathrm{RI}_{g(i+1)}^{\prime}}{\mathrm{RI}_{g(i)}^{\prime}}
$$

Combining expressions 21, 24, and 25, we have

$$
\frac{1}{2} \ln \frac{\mathrm{EI}_{g(i+1)}}{\mathrm{EI}_{g(i)}} \approx \frac{1}{2} \ln \frac{\tilde{\mathrm{RI}}_{g(i+1)}}{\tilde{\mathrm{RI}}_{g(i)}} \approx \frac{1}{2} \ln \frac{\mathrm{EI}_{g(i+1)} \eta_{i+1}}{\mathrm{EI}_{g(i)} \eta_{i}},
$$

with $\eta_{i}=\cos ^{4(r+2)} \varphi_{i} \beta_{i}^{4 \tan ^{2} \varphi(r+2)}$ representing the difference between $\mathrm{RI}_{g(i)}^{\prime}$ and $\mathrm{RI}_{g(i)}$. The estimated ray impedance of rigidity is

$$
\tilde{\mathrm{RI}}_{g(i)} \approx \mathrm{EI}_{g(i)} \eta_{i}
$$

In Figure 8, the Rg curves represented by $\mathrm{EI}_{g}$ and $\mathrm{RI}_{g}$ have different deviations from the real $\mathrm{Rg}$ curves. The reflectivity derived from $\tilde{\mathrm{RI}}_{g}$ agrees with the $R_{g}$ represented by $\mathrm{EI}_{g}$ over the full range of angles of incidence. Combining expressions 18 and 27, the estimated ray impedance from elastic impedance is

$$
\tilde{\mathrm{RI}}_{i}\left(p\left(\theta_{i}\right)\right)=\tilde{\mathrm{RI}}_{f(i)} \cdot \tilde{\mathrm{RI}}_{g(i)} \approx \mathrm{EI}_{i} \frac{\alpha^{-\tan ^{2} \theta} \eta_{i}}{\cos \theta_{i}},
$$

where $\alpha$ is the average velocity of the section of interest, $\eta_{i}=\cos ^{4(r+2)} \varphi_{i} \beta_{i}^{4 \tan ^{2} \varphi(r+2)}, \varphi_{i}$ is the angle of shear reflection at the $i$ th interface $\left(\sin \varphi_{i}=\frac{\beta_{i}}{\alpha_{i}} \sin \theta_{i}\right)$. R $\tilde{I}_{i}\left(p\left(\theta_{i}\right)\right)$ represents the elastic property of a medium within a certain depth/time interval. It is based on the same physical property as EI, but has similar interpretation capability to RI.

The analysis of this estimate is still performed in the angle domain using three single-interface models, to retain the physical meaning of all three measurements. EI and RI are estimated with the real value of parameters $K$ and $r$, respectively. In Figure 9, after the transformation from EI curves using expression 28, RI curves have comparable value range to $\mathrm{AI}$, and a similar variation as $\mathrm{RI}$ curves with increasing incident angle. Therefore, the introduced formula can be used as an approximate normalization of EI. Figure 10 compares the reflection coefficients approximated by EI, RI, and $\tilde{\mathrm{RI}}$ with other approximations of Figure 1. The estimated RI does not compromise the accuracy of original EI in approximating reflection coefficients. The reflection coefficients calculated from RI (Figure 10) agree well with that of EI over the range of precritical angle for all three models, and thus show better accuracy than that of original RI. In Models 2 and 3, the reflectivities represented by EI deviate from the exact Zoeppritz equation and other approximations in the regions of large angle of incidence (larger than $50^{\circ}$ ). However, this defect is overcome by the estimation of new RI, whose reflection coefficients agree with exact ones even at the postcritical angle.

\section{REAL LOG DATA ANALYSIS}

We first use real log data for three different types of reservoirs to evaluate the interpretation capability of elastic impedance and ray impedance, and test the feasibility of estimating RI from EI. Crossplots of EI, RI, and RI at large angles of incidence versus AI are plotted for lithology discrimination. A linear/quadratic discrimination analysis is applied to quantify the performance of the crossplots using the three impedance approximations. The RI of a 
real seismic section is estimated based on the inverted EI section for the tight-sand gas reservoir, and is compared with the inverted RI.

Case 1: This reservoir formation is a traditional Class III gassand (Avseth et al., 2005), which has high porosity (>25\%) and permeability. Figure 11 compares the logs of AI, EI, RI, and RI, and the crossplots between three form elastic impedance and
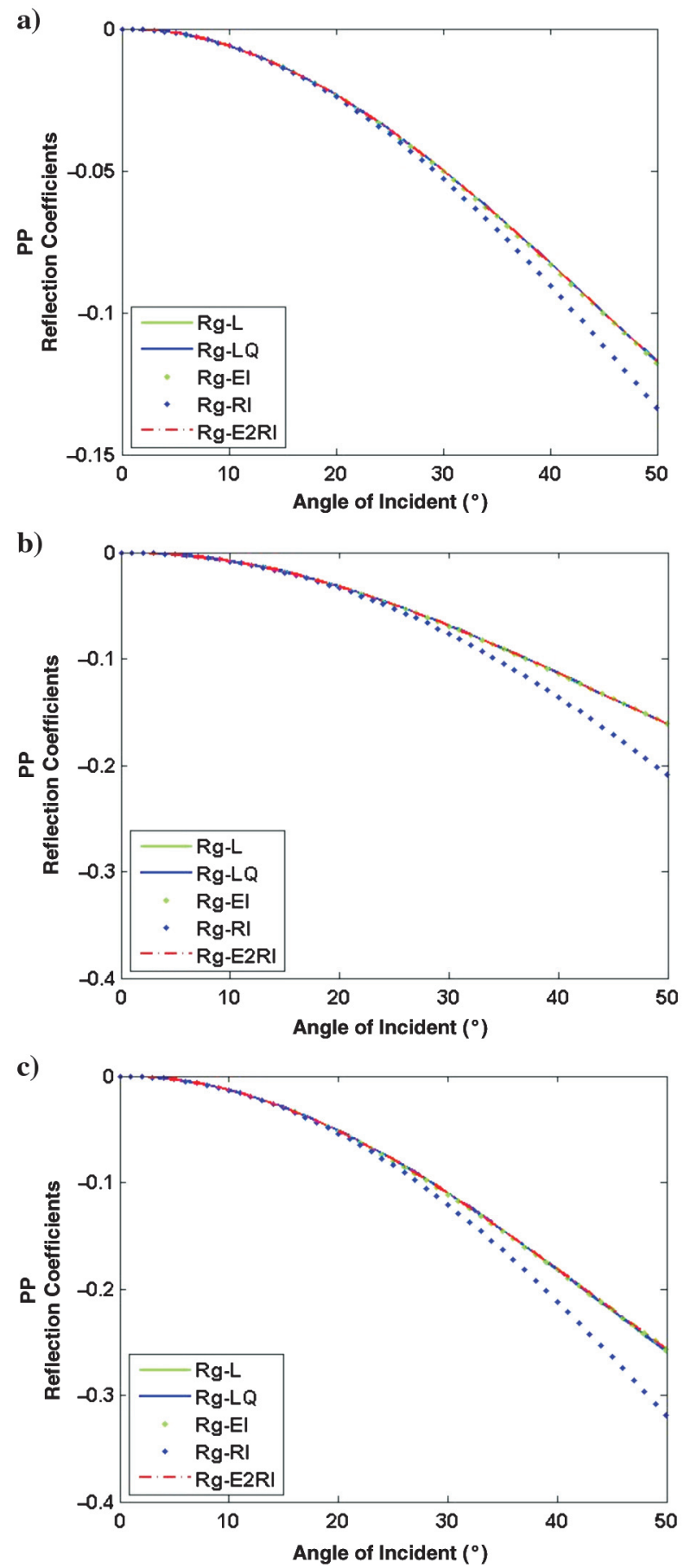

Figure 8. Comparison of RI, EI, and R̃ for (a) Model 1, (b) Model 2, and (c) Model 3: Solid curves are RI. Dashed curves represent EI and RI, respectively. AIs are also plotted as the straight dotted lines. The overburden shale layers and lower sand layers are blue and red, respectively. acoustic impedance. The gamma ray log is used for lithology identification. In the crossplots, RI $(250 \mathrm{~ms} / \mathrm{km})$ and EI $\left(49^{\circ}\right)$ give a similar characterization of lithology (Figure 11b and 11d). Both of them show good discrimination of sand from shale. EI has a value
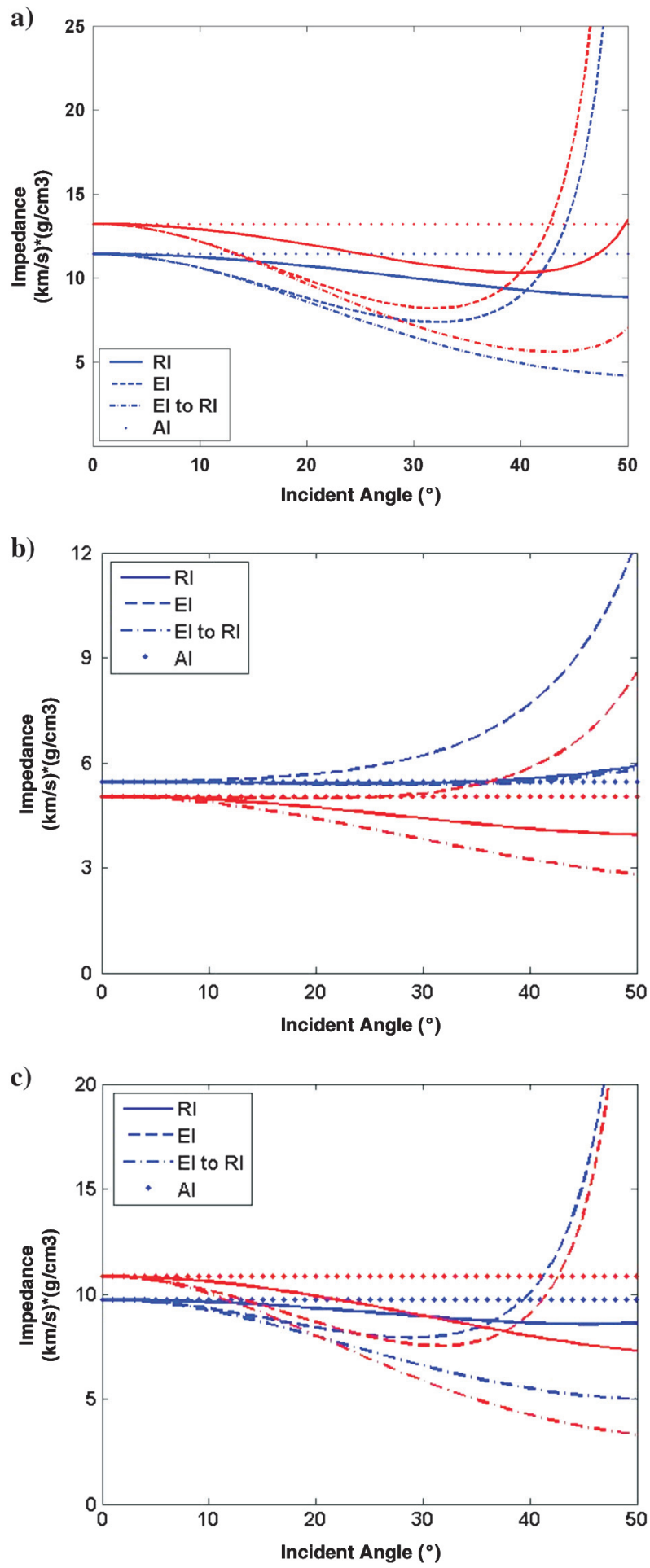

Figure 9. Comparison of RI, EI, and R̃ for (a) Model 1, (b) Model 2, and (c) Model 3: Solid curves are RI. Dashed curves represent EI and RI, respectively. AIs are also plotted as the straight dotted lines. The overburden shale layers and lower sand layers are blue and red, respectively. 

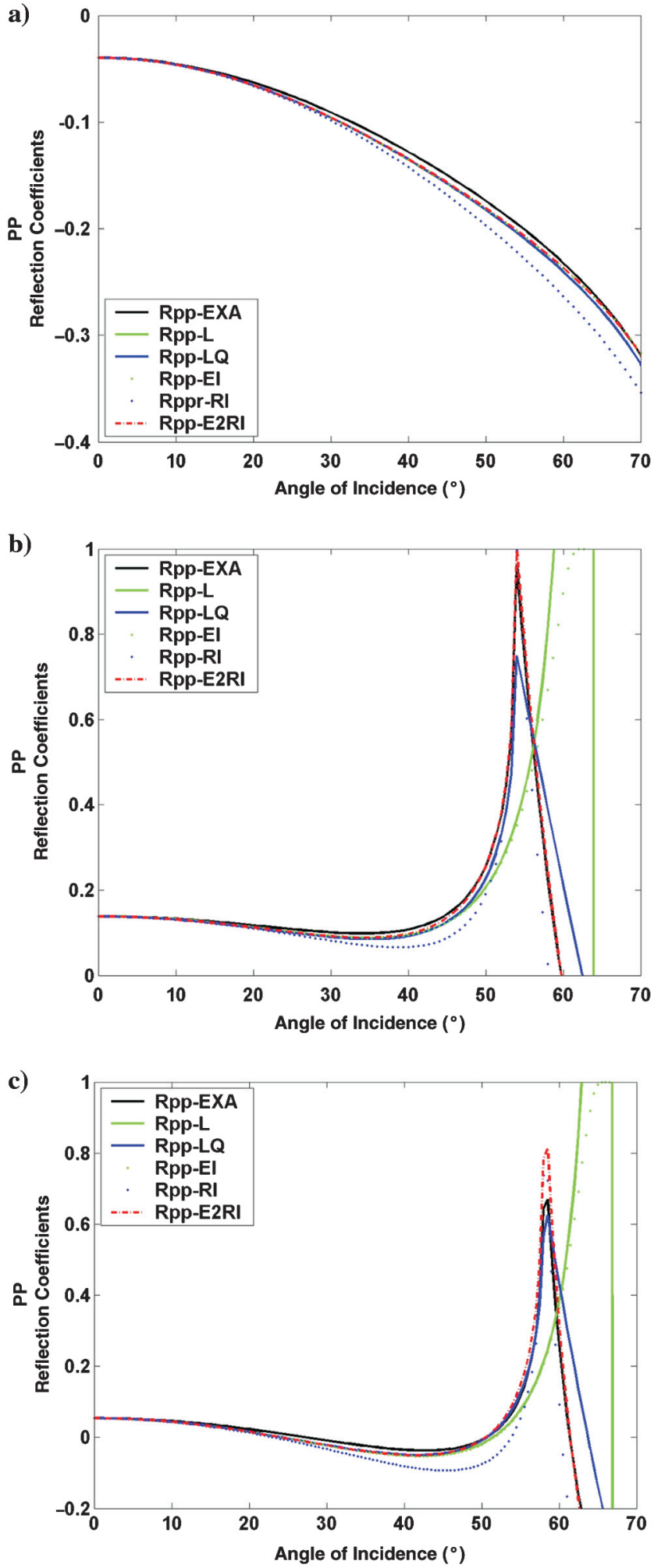

Figure 10. Comparison of different forms of AVO curves for (a) Model 1, (b) Model 2, and (c) Model 3. Black curves represent the reflection coefficients from exact Zeoppritz equation. The solid green and blue curves represent the linearized approximations from equations 7 and 8, respectively; the dotted green and blue lines, respectively, show the approximations calculated from EI (equation 3) and RI (equation 5). Dashed red curves are the reflectivity represented by RI. range of three times that of RI; RI has a range comparable to RI and AI. The transformation also retains the interpretation ability of EI/RI: a clear view of sand can also be found in the crossplot of RI (Figure 11f). Based on the linear discrimination analysis (Figure 9c, 9e, and 9f), the misclassification error rate of three crossplots is $24.9 \%, 31.0 \%$, and $26.9 \%$, respectively.

Case 2: This Class I gas-sand is an unconventional resource with tight clastic sediments showing extremely low porosity (average $2.9 \%$ ) and permeability (average $3.9 \mathrm{mD}$ ). In this case, sands are mixed with shale and thus are difficult to be discriminated from the surrounding shales. The ray impedance $(150 \mathrm{~ms} / \mathrm{km})$ shows a better interpretation of the reservoir than the elastic impedance (Figure 12b-12e). A quadratic discrimination analysis shows that the misclassification error rate of using RI and EI is $19.2 \%$ and $27.7 \%$, respectively. This performance is recovered by the estimation of RI from elastic impedance (Figure $12 \mathrm{f}$ and $12 \mathrm{~g}$ ), with the misclassification error rate of $22.5 \%$.

Case 3: The last analysis is performed on a reservoir of multiple thin oil-bearing sand layers (sand-mud interbedded layers). The porosity and permeability are again low, ranging from $10.4 \%$ to $13.9 \%$ and from $0.06 \mathrm{mD}$ to $14.7 \mathrm{mD}$, respectively. In this case, although good discrimination of sand is found in the crossplots of RI and EI against $\mathrm{AI}$ (with respective misclassification error rate of $9 \%$ and $13.6 \%$ ), EI has the value almost twice of that of RI. After the transformation using equation 28 , the RI then has a similar performance (with misclassification error rate of $11.1 \%$ ) and range of value as the original RI.

\section{APPLICATION ON REAL SEISMIC DATA}

$\mathrm{EI}\left(49^{\circ}\right)$ and $\mathrm{RI}(150 \mathrm{~ms} / \mathrm{km})$ sections are inverted using the seismic data from the tight-sand reservoir. The study area is in Western Sichuan basin, southwest China, with the tight-sand gas deposits buried at around $5000 \mathrm{~m}$ in depth. According to the petrophysical analysis, the tight clastic sediments have extremely low porosity and permeability: most of the sand groups have a porosity less than $3 \%$ and the permeability is in the range from 0.02 to 0.08 milliDarcy. The major productive reservoir types are pores or fractures-plus-pores (Tang et al., 2008).

Among a series of Upper Triassic formations, the deepest member encountered by drilling is TX2 (Gan et al., 2009), which is the target of this study. It is a deposit of sand-mud alternating delta facies at a depth from 4500 to $5300 \mathrm{~m}$. This formation consists of seven groups of gas-bearing sand layers. The variation of the fluid properties does not lead to significant changes either in the elastic parameters or in the seismic response. In fact, based on the analysis of well logs, there is little impedance difference between the sand and shale, which is a challenge to the seismic inversion.

We perform the ray impedance inversion in the following steps (Lu and Wang, 2009): (1) Prestack time migration is applied to generate common image point (CIP) gathers; (2) Transforming CIP gathers from the offset domain to the ray-parameter domain by ray tracing; (3) Construction of constant ray parameter (CRP) profiles with selected P-values; (4) Mixed-phase wavelet estimation using a high-order statistic method on each CRP profile; (5) Performing reflection coefficients inversion through a model-constrained sparse-spike method; (6) Ray-impedances inversion from the reflectivity profiles.

The well-tie result is shown in Figure 14. Corresponding well logs and a related horizon are plotted on Figure 14 as well 
as inverted sections in Figure 15. Compared with inverted EI, gasbearing sand layers in the RI section show clearer contrast from surrounding rocks (higher impedance regions at well location) and therefore can be more easily identified. This property is recovered when we estimate the corresponding R̃ using equation 28 . A

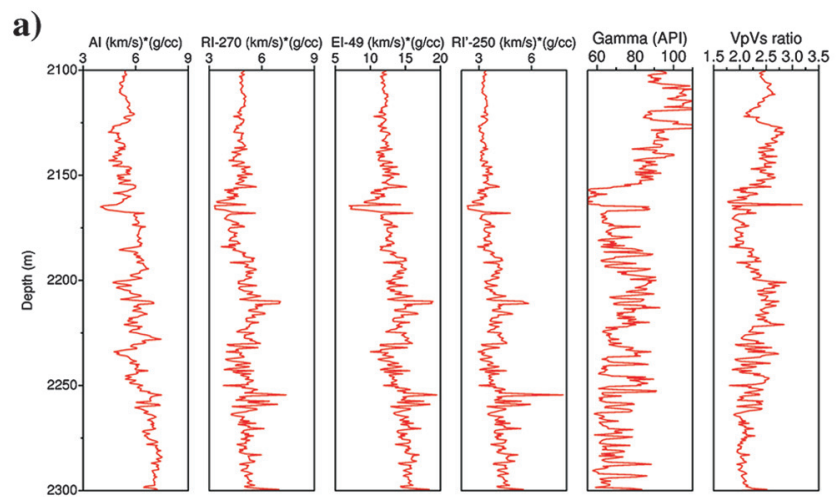

b)
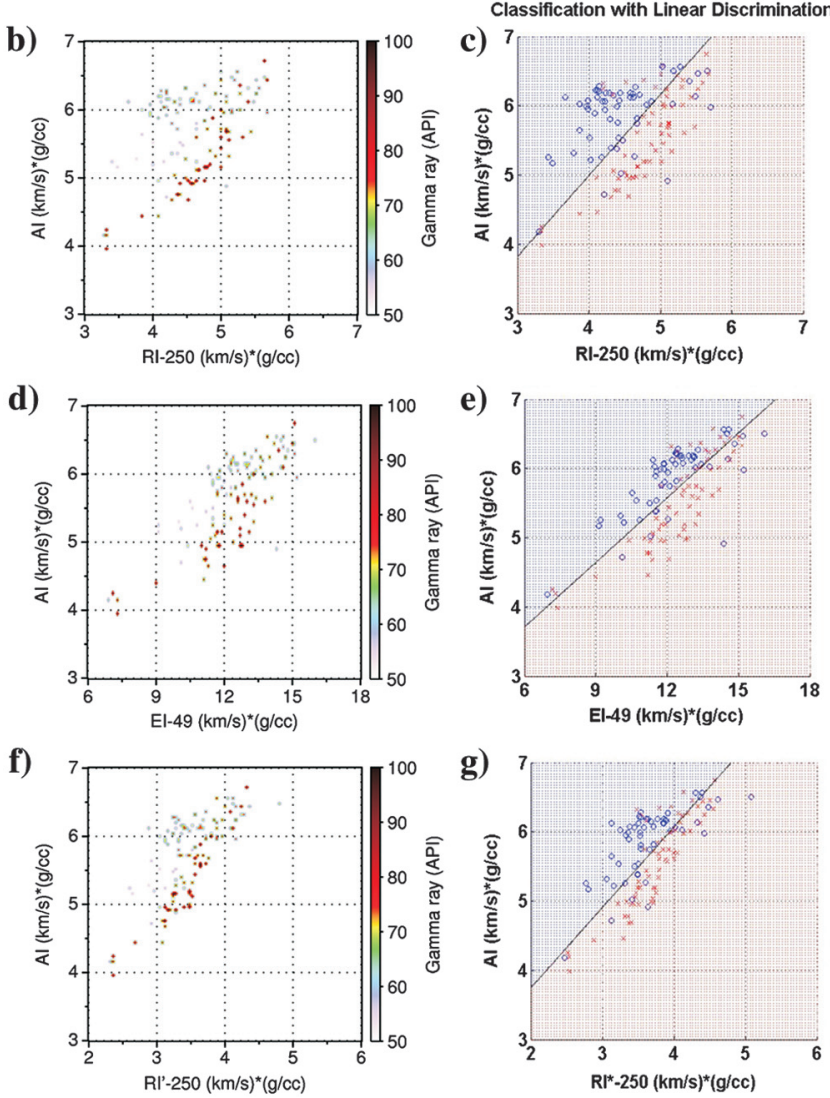

Figure 11. (a) Comparison of AI with RI (270 ms $/ \mathrm{km})$, EI $\left(48^{\circ}\right)$, and RI $(270 \mathrm{~ms} / \mathrm{km})$ in case 1 . (b, d, and $\mathrm{f})$ Crossplots of RI $(270 \mathrm{~ms} / \mathrm{km}), \mathrm{EI}\left(48^{\circ}\right)$ and RI $(270 \mathrm{~ms} / \mathrm{km})$ against AI in case 1. The color key represents the gamma ray log, with sands corresponding to lower values. (c, e, and g) Results of linear discrimination analysis for the crossplots. Hydrocarbon saturated sands with and shales are classified, respectively, as blue and red sections based on the gamma ray log with a threshold of 70API. The black line shows the classification boundary between two lithologies. The misclassification error rate of three crossplots is $19.2 \%, 27.7 \%$, and $22.5 \%$, respectively. shear-wave model is estimated using simultaneous inversion prior to this transformation. Regions with higher impedance values are more distinct after this transformation. Beside, the values of impedance are compressed within the range between 2-10 $(\mathrm{km} / \mathrm{s}) *(\mathrm{~g} / \mathrm{cc})$.

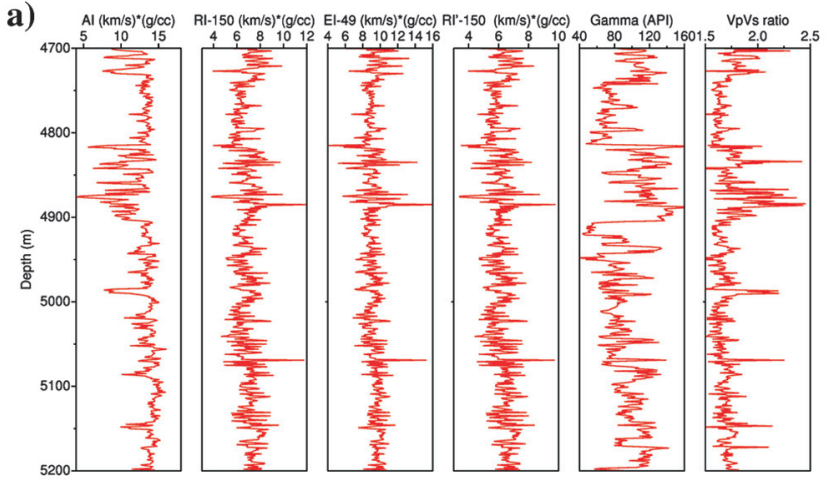

b)
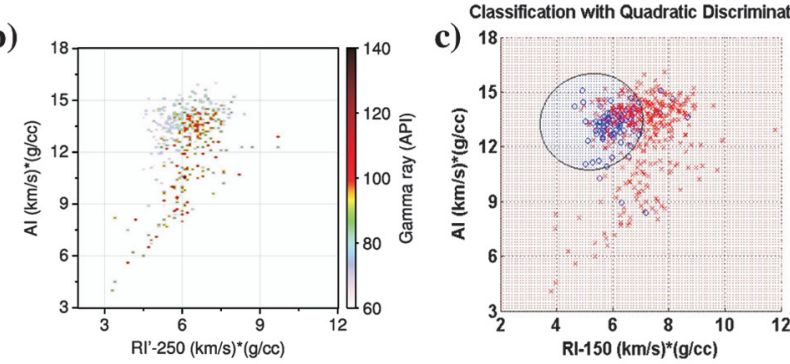

d)
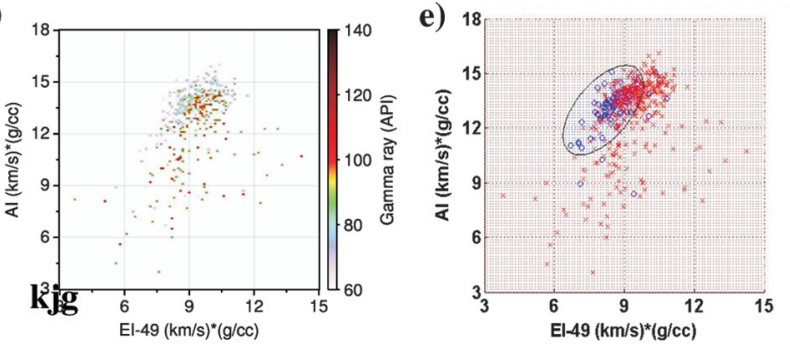

f)
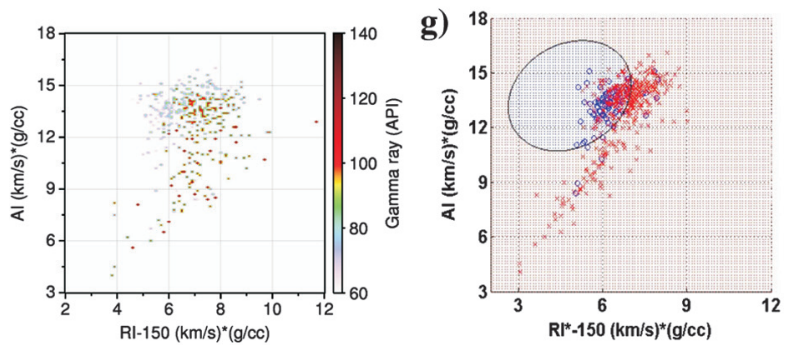

Figure 12. (a) Comparison of AI with RI $(150 \mathrm{~ms} / \mathrm{km})$, EI $\left(49^{\circ}\right)$, and RI $(150 \mathrm{~ms} / \mathrm{km})$ in case 2. (b, d, and f) Crossplots of RI $(150 \mathrm{~ms} / \mathrm{km}), \mathrm{EI}\left(49^{\circ}\right)$, and $\tilde{\mathrm{RI}}(150 \mathrm{~ms} / \mathrm{km})$ against $\mathrm{AI}$ in case 2 . The Color key represents the gamma ray log, with sands corresponding to lower values. (c, e, and g) Results of quadratic discrimination analysis for the crossplots. Sand saturated with hydrocarbons and shales are classified, respectively, as blue and red section based on the gamma ray log with a threshold of 70API. The black circle shows the boundary equation of two sections. The misclassification error rate of three crossplots is $24.9 \%$, $31.0 \%$, and $26.9 \%$, respectively. 


\section{DISCUSSIONS}

The elastic impedance and ray impedance represent elastic properties for subsurface media along different paths. The elastic impedance assumes a constant angle of incidence, whereas the ray impedance is defined along the wave propagation ray path, considering various angles. Although Aki and Richards' well-known

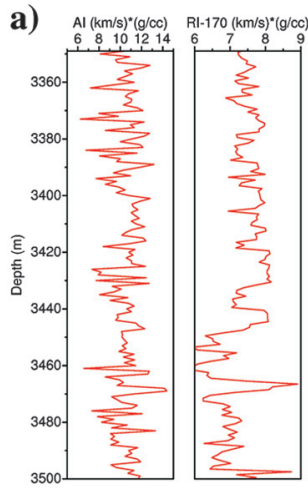

b)

d)

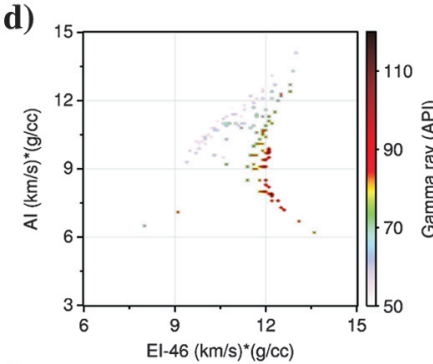

f)

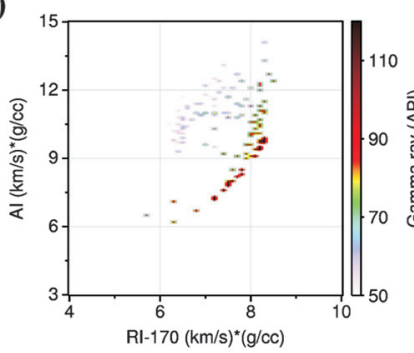

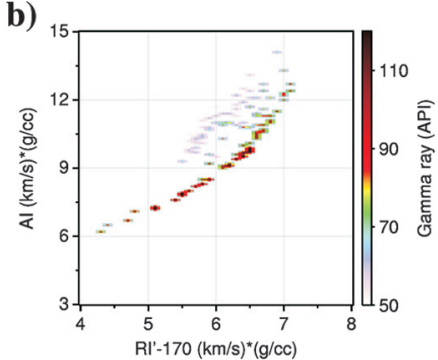

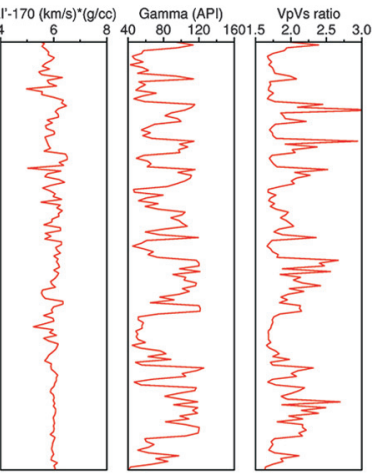

c) Classification with Linear Discrimination
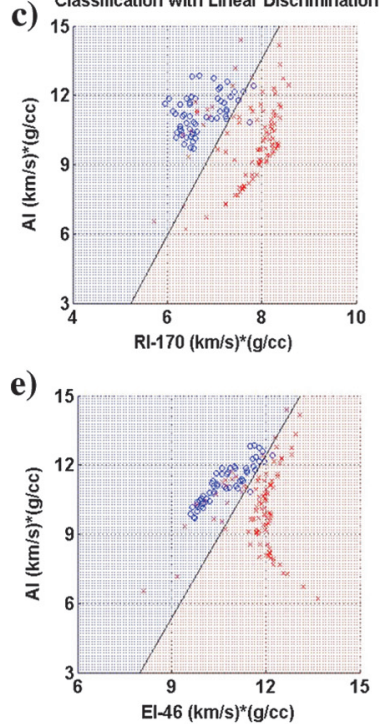

g)

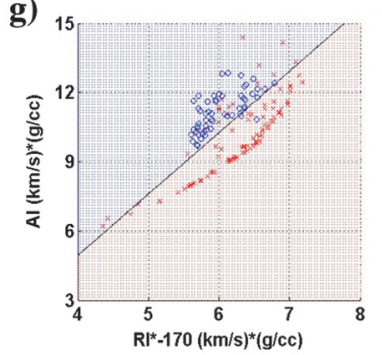

Figure 13. (a) Comparison of AI with RI (170 ms $/ \mathrm{km})$, EI $\left(46^{\circ}\right)$, and RI $(170 \mathrm{~ms} / \mathrm{km})$ in case 3 . (b, d, and f) Crossplots of RI $(170 \mathrm{~ms} / \mathrm{km})$, EI $\left(46^{\circ}\right)$, and RI $(170 \mathrm{~ms} / \mathrm{km})$ against AI in case 3 . The color key represents the gamma ray log, with sands corresponding to lower values. (c, e, and g) Results of quadratic discrimination analysis for the crossplots. Hydrocarbon saturated sand and shales are classified, respectively, as blue and red section based on the gamma ray log with a threshold of 65API. The black line shows the boundary equation of two sections. The misclassification error rate of three crossplots is $9 \%, 13.6 \%$, and $11.1 \%$, respectively. approximation of the reflection coefficient (equation 7) is limited to represent the subsurface property at very wide angles of incidence, good accuracy of approximating this reflectivity is preserved in the derivation of EI, which is able to discriminate a typical Class III gas-sand from surrounding rocks. Theoretical analysis shows that at small or middle angles of incidence, an EI curve has variation similar to RI within the local depth interval. EI without normalization has a dramatically varying value at large angles of incidence, but large variation in the values alone is not a

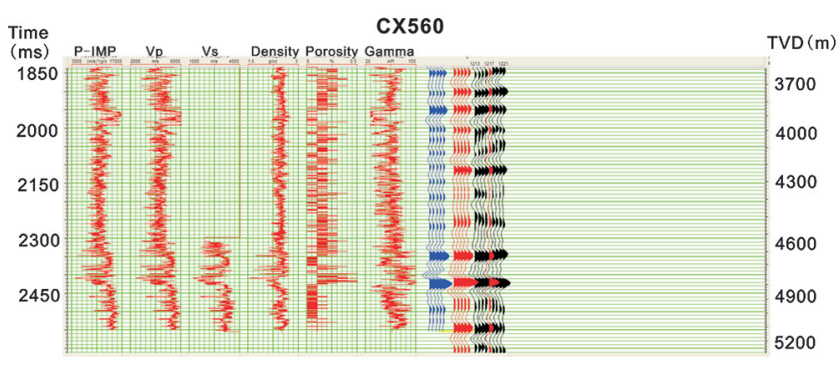

Figure 14. Calibration result of well logs and seismic data. Six curves in red are the well logs of acoustic impedance, P-wave velocity, S-wave velocity, density, porosity, and gamma ray. Traces in blue are five repetitive synthetic traces generated using the well logs and wavelet estimates. Traces in red are five repetitive seismic traces (black) at the well location.
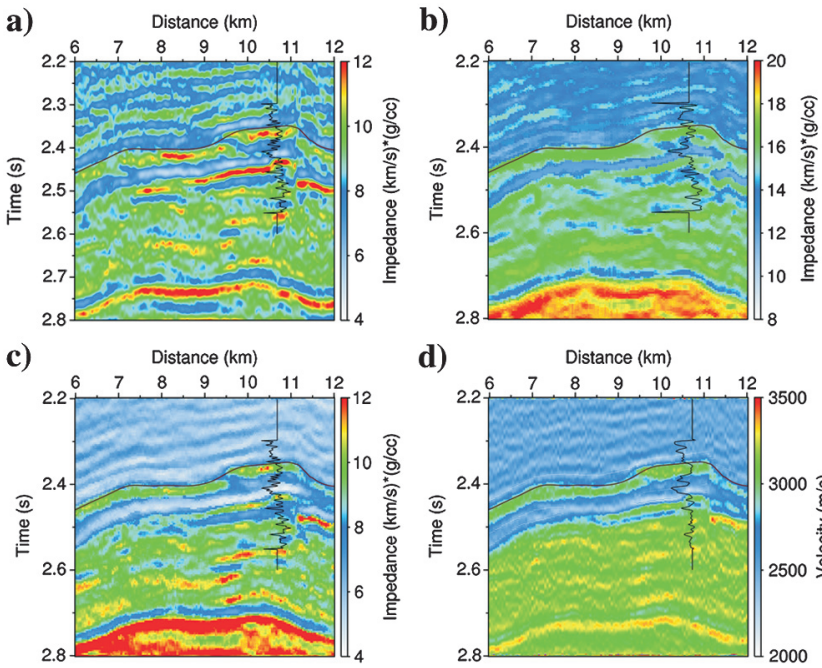

d) Distance $(\mathrm{km})$
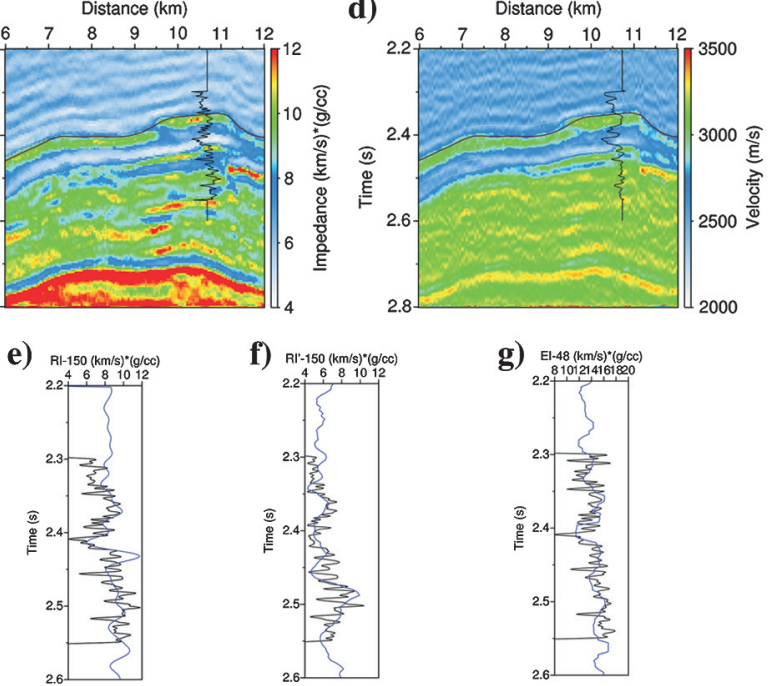

Figure 15. Inverted section of (a) RI (150 ms $/ \mathrm{km})$, (b) EI $\left(49^{\circ}\right)$ and (c) RI $(150 \mathrm{~ms} / \mathrm{km})$ for the reservoir in case 2 . (d) Shear-wave velocity model used for transformation. At the well location, the inverted traces (blue curves) are compared with their corresponding logs (black curves). 
problem, especially for the lithology interpretation using crossplots. However, due to the significant varying value range, inaccurate values of $K$ have a great influence on the absolute values of EI. They change not only the relative relationship between EIs of different subsurface layers but also the contrast between EI and AI. Therefore, certain correction procedures (optimization of $K$ and normalization of EI) are necessary when EI is used in reservoir characterization. Ray impedance can be used to describe continuous media within an arbitrary interval. Because of its stable value range and nonsensitivity to errors, ray impedance is more applicable than elastic impedance for reservoir characterization.

In the evaluation of the crossplots of three attributes, the linear/ quadratic discrimination analysis needs a gamma ray threshold, which can be set according to the lithology interpretation. Although the misclassification error rate depends on the threshold, a different threshold does not change the relative error rate of the crossplots among three attributes. The introduced RI shows good accuracy in representing reflectivities and retains similar interpretation capability of original RI. However, in practice, the transformation of RI from EI requires a background shear-wave velocity model. The model can be built by the extrapolation of the low-pass filtering well $\log$ along the horizons, or inverted from seismic data. The simultaneous inversion is now a routine technique, which provides more accurate results updated from the smooth initial model. In the application of real data, we use five PP-wave seismic sections with constant ray parameters to estimate the P-wave velocity, S-wave velocity, and density, simultaneously.

\section{CONCLUSIONS}

In this paper, beside the evaluation of the merits and drawbacks of conventional EI and RI, we propose a new measurement RI estimated from EI by overcoming the disadvantages of EI and RI. The introduced RI has more appropriate accuracy in approximating reflectivity than EI and RI. RI also solves the range variability problem of original EI and has a similar good performance of interpretation as the RI. In this sense, the introduced RI can be used as an appropriate normalization of EI.

In the application of well log data, although crossplots of EI against AI show visually similar performance as those of ray impedance (RI), larger misclassification error rates are found in the linear/quadratic discrimination analysis. RI estimated from EI has improved discrimination capabilities of lithologies compared to original EI. The transformation from EI to RI also acts as normalization. Finally, we estimate the RI from the inverted EI section for the tight-sand gas reservoir. A shear-wave model is estimated prior to this transformation. The inverted result is improved during this transformation because the gas-bearing sand layers with higher impedance values in the new RI section can be more easily identified. Therefore, in practice, estimation of RI from existing EI by means of the introduced transformation could straightforwardly improve the inversion result.

\section{ACKNOWLEDGMENTS}

We are grateful to the sponsors of the Center for Reservoir Geophysics, Imperial College London, and the Edinburgh Anisotropy Project, British Geological Survey for supporting this research. Feng Zhang is also sponsored by the research funds provided to new recruitments of China University of Petroleum-Beijing (YJRC-2011-03), CNPC Geophysical New Method and
Technology Project (Geophysical Methods for Unconventional Resources) and the Major National Science and Technology Project under the contract of 2011ZX05019008. Acknowledgement also goes to editors and anonymous reviews for their constructive review.

\section{APPENDIX A}

\section{CONVENTIONAL ELASTIC IMPEDANCE}

The definition of the elastic impedance was proposed by Connolly (1999). At the $i$ th interface, the linearized approximation for PP-wave reflection coefficients (Aki and Richards, 1980) is

$$
\begin{aligned}
R_{L(i)}(\theta) \approx & \frac{1}{2}\left(1+\tan ^{2} \theta\right) \frac{\Delta \alpha}{\alpha}-4 \frac{\beta^{2}}{\alpha^{2}} \sin ^{2} \theta \frac{\Delta \beta}{\beta} \\
& +\left[\frac{1}{2}-2 \frac{\beta^{2}}{\alpha^{2}} \sin ^{2} \theta\right] \frac{\Delta \rho}{\rho},
\end{aligned}
$$

where $\quad \alpha=\frac{\alpha_{2}+\alpha_{1}}{2}, \quad \beta=\frac{\beta_{2}+\beta_{1}}{2}, \quad \rho=\frac{\rho_{2}+\rho_{1}}{2}, \quad \Delta \alpha=\alpha_{2}-\alpha_{1}$, $\Delta \beta=\beta_{2}-\beta_{1}$, and $\Delta \rho=\rho_{2}-\rho_{1}$. The reflectivity is defined in the recursive form in Connolly (1999)

$$
R_{L(i)}(\theta)=\frac{\mathrm{EI}_{i+1}(\theta)-\mathrm{EI}_{i}(\theta)}{\mathrm{EI}_{i+1}(\theta)+\mathrm{EI}_{i}(\theta)} \approx \frac{1}{2} \ln \frac{\mathrm{EI}_{i+1}(\theta)}{\mathrm{EI}_{i}(\theta)}
$$

where $\mathrm{EI}_{i}$ and $\mathrm{EI}_{i+1}$ are the elastic impedance above and below the interface, respectively. Equation A1 can be expressed as

$$
R_{L(i)}(\theta) \approx \frac{1}{2} \ln \frac{\alpha_{i+1}^{1+\tan ^{2} \theta} \beta_{i+1}^{-8 K \sin ^{2} \theta} \rho_{i+1}^{1-4 K \sin ^{2} \theta}}{\alpha_{i}^{1+\tan ^{2} \theta} \beta_{i}^{-8 K \sin ^{2} \theta} \rho_{i}^{1-4 K \sin ^{2} \theta}},
$$

with $\frac{\Delta \alpha}{\alpha} \approx \ln \frac{\alpha_{i+1}}{\alpha_{\mathrm{i}}}, \frac{\Delta \beta}{\beta} \approx \ln \frac{\beta_{i+1}}{\beta_{i}}, \frac{\Delta \rho}{\rho} \approx \ln \frac{\rho_{i+1}}{\rho_{i}}$, and $K=\beta^{2} / \alpha^{2}$. It is possible to represent $R_{L(i)}(\theta)$ recursively, only if $\theta_{i} \approx \theta$ is constant, and $K$ is constant. Therefore the angle-dependent elastic impedance is

$$
\mathrm{EI}_{i}=\alpha_{i} \rho_{i}\left(\alpha_{i}^{\tan ^{2} \theta} \beta_{i}^{-8 K \sin ^{2} \theta} \rho_{i}^{-4 K \sin ^{2} \theta}\right)
$$

\section{APPENDIX B}

\section{DERIVATION OF RAY IMPEDANCE}

This appendix is referenced from Wang (2003), and presented here for the sake of completeness of this current paper. The ray impedance can be derived from the linearized form of quadratic reflectivity (Wang, 1999)

$$
R_{L Q}(p) \approx \mathrm{Rf}-2 \frac{\Delta \mu}{\rho} p^{2}
$$

where $\mathrm{Rf}$ is the fluid-fluid term determined by the acoustic impedance along the wave propagation ray path. At the $i$ th interface, 


$$
\begin{aligned}
\operatorname{Rf}_{i}\left(p\left(\theta_{i}\right)\right) & =\frac{\rho_{i+1} q_{\alpha \mathrm{i}}-\rho_{i} q_{\alpha(\mathrm{i}+1)}}{\rho_{i+1} q_{\alpha \mathrm{i}}+\rho_{i} q_{\alpha(\mathrm{i}+1)}} \\
& =\frac{1}{2} \ln \left(\frac{\alpha_{i+1} \rho_{i+1}}{\cos \theta_{\mathrm{i}+1}} / \frac{\alpha_{i} \rho_{i}}{\cos \theta_{\mathrm{i}}}\right)
\end{aligned}
$$

where $q_{\alpha(i)}=\sqrt{1 / \alpha_{i}^{2}-p^{2}}$ and $q_{\alpha(i+1)}=\sqrt{1 / a_{i+1}^{2}-p^{2}}$ are the $\mathrm{P}$-wave vertical slownesses. The rigidity term is

$$
\operatorname{Rg}_{i}\left(p\left(\varphi_{i}\right)\right)=-2 \frac{\Delta \mu}{\rho} p^{2} \approx-2\left(\frac{\Delta \rho}{\rho}+2 \frac{\Delta \beta}{\beta}\right) \sin ^{2} \varphi,
$$

where $\varphi_{i}$ is the angle of conversion at the $i$ th interface, $\varphi$ is the average angle of conversion above and below the interface $\Delta \mu=\rho_{i+1} \beta_{i+1}^{2}-\rho_{i} \beta_{i}^{2} \approx \beta^{2} \Delta \rho+2 \rho \beta \Delta \beta$ and $p=\sin \varphi / \beta$. Assuming $\frac{\Delta \rho}{\rho} \approx r \frac{\Delta \beta}{\beta}$ and the conversion angles are smaller than $30^{\circ}$, the above expression is modified to

$$
\operatorname{Rg}_{i}\left(p\left(\varphi_{i}\right)\right) \approx-2(r+2) \frac{\Delta \beta}{\beta} \tan \varphi^{2}
$$

If using the second order Taylor's series expansions to approximate Snell's law

$$
\frac{\alpha_{i}}{\alpha_{i+1}}=\frac{\sin \theta_{i}}{\sin \theta_{i+1}}
$$

we have

$$
\frac{\alpha_{i}}{\alpha_{i+1}} \approx 1-\frac{\Delta \alpha}{\alpha}+\frac{1}{2}\left(\frac{\Delta \alpha}{\alpha}\right)^{2}
$$

and

$$
\begin{aligned}
\frac{\sin \theta_{i}}{\sin \theta_{i+1}} & \approx 1-2 \frac{\Delta \sin \theta}{\sin \theta}+2\left(\frac{\Delta \sin \theta}{\sin \theta}\right)^{2} \\
& =1-2 \frac{\tan \frac{\Delta \theta}{2}}{\tan \theta}+2\left(\frac{\tan \frac{\Delta \theta}{2}}{\tan \theta}\right)^{2},
\end{aligned}
$$

where $\theta=\frac{\theta_{i+1}+\theta_{i}}{2}$ and $\Delta \theta=\theta_{i+1}-\theta_{i}$. A quadratic equation is built by combining equations $\mathrm{B} 5, \mathrm{~B} 6$, and $\mathrm{B} 7$

$$
\left(\frac{\tan \frac{\Delta \theta}{2}}{\tan \theta}\right)^{2}-\frac{\tan \frac{\Delta \theta}{2}}{\tan \theta}+\frac{1}{2} \frac{\Delta \alpha}{\alpha}-\frac{1}{4}\left(\frac{\Delta \alpha}{\alpha}\right)^{2} \approx 0 .
$$

One of the solutions of the above equation is

$$
\tan \frac{\Delta \theta}{2} \approx \frac{1}{2} \tan \theta \frac{\Delta \alpha}{\alpha} .
$$

Following Snell's law, $\theta$ and $\alpha$ can be substituted, respectively, by $\varphi$ and $\beta$

$$
\tan \frac{\Delta \varphi}{2} \approx \frac{\Delta \varphi}{2} \approx \frac{1}{2} \tan \varphi \frac{\Delta \beta}{\beta} .
$$

The rigidity term is derived by

$$
\begin{aligned}
\operatorname{Rg}_{i}\left(p\left(\varphi_{i}\right)\right) & \approx-2(r+2) \Delta \varphi \tan \varphi \approx 2(r+2) \frac{\Delta \cos \varphi}{\cos \varphi} \\
& \approx \frac{1}{2} \ln \frac{\cos ^{4(r+2)} \varphi_{i+1}}{\cos ^{4(r+2)} \varphi_{i}},
\end{aligned}
$$

where $\varphi=\frac{\varphi_{i+1}+\varphi_{i}}{2}$ and $\Delta \varphi=\varphi_{i+1}-\varphi_{i}$. The reflectivity at the $i$ th interface is now expressed as

$$
\begin{aligned}
\operatorname{Rg}_{i}\left(p\left(\theta_{i}, \varphi_{i}\right)\right) & \approx \frac{1}{2} \ln \frac{\mathrm{RI}_{i+1}\left(\theta_{i+1}, \varphi_{i+1}\right)}{\mathrm{RI}_{i}\left(\theta_{i}, \varphi_{i}\right)} \\
& =\frac{1}{2} \ln \left(\frac{\alpha_{i+1} \rho_{i+1} \cos ^{4(r+2)} \varphi_{i+1}}{\cos \theta_{i+1}} / \frac{\alpha_{i} \rho_{i} \cos ^{4(r+2)} \varphi_{i}}{\cos \theta_{i}}\right) .
\end{aligned}
$$

Therefore, the ray impedance is defined as (Wang, 2003)

$$
\mathrm{RI}_{i}\left(\theta_{i}, \varphi_{i}\right)=\frac{\alpha_{i} \rho_{i} \cos ^{4(r+2)} \varphi_{i}}{\cos \theta_{i}}
$$

\section{REFERENCES}

Aki, K., and P. G. Richards, 1980, Quantitative seismology: Theory and methods: W. H. Freeman.

Avseth, P., T. Mukerji, and G. Mavko, 2005, Quantitative seismic interpretation: Cambridge University Press.

Bortfeld, R., 1961, Approximation to the reflection and transmission coefficients of plane longitudinal and transverse waves: Geophysical Prospecting, 9, 485-502, doi: 10.1111/gpr.1961.9.issue-4.

Connolly, P., 1999, Elastic impedance: The Leading Edge, 18, 438-452, doi: 10.1190/1.1438307.

Gan, Q., D. Xu, J. Tang, and Y. H. Wang, 2009, Seismic resolution enhancement for tight-sand gas reservoir characterization: Journal of Geophysics and Engineering, 6, 21-28, doi: 10.1088/1742-2132/6/1/002.

Hilterman, F. J., 2001, Seismic amplitude interpretation: 2001 distinguished instructor short course, No. 4, SEG.

Lu, X., and Y. Wang, 2009, Elastic parameters inversion through ray impedance: 71st Annual International Conference and Exhibition, EAGE, Extended Abstracts, S023.

Rutherford, S. R., and R. H. Williams, 1989, Amplitude-versus-offset variations in gas sands: Geophysics, 54, 680-688, doi: 10.1190/1.1442696.

Santos, L. T., and M. Tygel, 2004, Impedance-type approximations of the P-P elastic reflection coefficients: Modeling and AVO inversion: Geophysics, 69, 592-598, doi: 10.1190/1.1707079.

Sava, P. C., and S. Fomel, 2003, Angle-domain common-image gathers by wavefield continuation methods: Geophysics, 68, 1065-1074, doi: 10 $.1190 / 1.1581078$.

Tang, J., et al., 2008, PP and PS seismic response from fractured tight gas reservoirs: A case study: Journal of Geophysics and Engineering, 5, 92102, doi: 10.1088/1742-2132/5/1/010.

VerWest, B., 2004, Elastic impedance revisited: 66th Annual International Conference and Exhibition, EAGE, Extended Abstracts, P342.

Wang, Y., 1999, Approximations to the Zoeppritz equations and their use in AVO analysis: Geophysics, 64, 1920-1927, doi: 10.1190/1.1444698.

Wang, Y., 2003, Seismic amplitude inversion in reflection tomography: Elsevier.

Whitcombe, D. N., 2002, Elastic impedance normalization: Geophysics, 67, 60-62, doi: 10.1190/1.1451331.

$\mathrm{Xu}$, S., H. Chauris, G. Lambare, and M. Noble, 2001, Common-angle migration: A strategy for imaging complex media: Geophysics, 66, 18771894, doi: 10.1190/1.1487131.

Zhang, F., 2010, Joint inversion of seismic PP- and PS-waves in the ray parameter domain: Ph.D. thesis, Imperial College London. 